\title{
Improvisation et processus compositionnel dans la genèse de Fenêtre Ovale de Karl Naëgelen
}

\section{Clément Canonne}

\begin{abstract}
Introduction
Même si le concept d'improvisation tend souvent à se définir par opposition au concept de composition-pour des raisons qui tiennent sans doute aux conditions historiques de leur cristallisation dans le monde occidental ${ }^{1}$-il serait cependant naïf de croire que ces deux manières de produire de la musique sont hermétiquement séparées dans la diversité de nos pratiques musicales. Comme l'a montré de manière convaincante Bruno Nettl (1974), il existe en fait un véritable continuum poïétique, qui nous fait passer insensiblement de l'improvisation à la composition ou de la composition à l'improvisation, de sorte que la frontière entre les deux reste en réalité toujours insaisissable (voir également Benson 2003). Le couple improvisation/composition ne fonctionne assurément pas comme une dichotomie qui partagerait clairement en deux mondes bien séparés l'ensemble de notre espace musical: il semble en effet y avoir à la fois des phénomènes qui empruntent simultanément à ces deux catégories (le raga indien), et des phénomènes qui ne se satisfont ni d'une description en termes de composition, ni d'une description en termes d'improvisation, comme l'organum médiéval (Dahlhaus 2010).
\end{abstract}

Mais l'opposition improvisation/composition, en plus d'être fragile conceptuellement, est également particulièrement inadaptée à rendre compte des multiples œuvres ou formes de pratiques musicales qui ont émergé depuis quelques décennies et qui semblent vouloir conjuguer composition et improvisation. L'improvisation s'est en effet trouvée mobilisée par la composition, soit implicitement—sous couvert de termes comme «musique aléatoire,» «indétermination» ou «œuvre ouverte»—chez des compositeurs comme John Cage, Earle Brown ou André Boucourechliev, soit explicitement, par exemple chez Vinko Globokar, Anthony Braxton ou John Zorn, sans oublier les tentatives faites en ce sens par les compositeurs du Third Stream (voir Lewis 1996; Globokar 1971). Dans tous les cas, l'interprétation de ces pièces requiert un acte d'improvisation, que ce soit en raison de l'indétermination intrinsèque de la notation utilisée (partitions graphiques ou verbales...), ou parce qu'il est explicitement demandé aux interprètes d'improviser plus ou moins librement à certains moments (à partir d'un réservoir d'accords ou de motifs, comme dans certaines pièces de Maurice Ohana, ou d'un ensemble de règles d'interaction, comme dans les game pieces de John Zorn).

En somme, qu'il s'agisse de renouveler la relation entre le compositeur et ses interprètes, d'aborder à nouveaux frais le rapport entre écriture et détermination ou encore d'affirmer la dimension intrinsèquement transculturelle de la production musicale contemporaine, cette hybridation de la composition et de l'improvisation n'a plus rien d'extraordinaire dans le monde de la musique savante occidentale, à tel point que l'on a pu proposer le néologisme de «comprovisation» pour désigner cet ensemble d'œuvres et de pratiques.

Ce vaste champ de la «comprovisation» constitue assurément un terrain d'investigation excitant pour qui entend réfléchir au rapport entre composition et improvisation, et à la manière dont ces deux facettes de la créativité musicale peuvent se conjuguer au sein d'un processus de création. Toutefois, l'éclosion de ces pratiques musicales hybrides ne doit pas nécessairement nous conduire à nier toute spécificité à l'improvisation comme à la composition: après tout, il ne peut y avoir hybridation sans différences spécifiques. ${ }^{3}$ Autrement dit, ce n'est pas parce qu'improvisation et composition se trouvent bien souvent inextricablement mêlées dans un certain nombre de phénomènes musicaux qu'il faut pour autant renoncer à identifier précisément ce qui fait la spécificité créative de ces deux manières de produire du musical. En particulier, il semble évident que les processus d'idéation ne fonctionnent pas de la même manière selon que l'on a affaire à une situation de composition ou à une situation d'improvisation, et ce en raison d'un rapport intrinsèquement différent à la temporalité qui vient moduler la manière dont s'opèrent les prises de décisions du musicien-les décisions du compositeur étant réversibles, souvent guidées par un plan ou une conception préalable, ${ }^{4}$ et résultant d'un choix délibéré, tandis que celles de l'improvisateur sont irréversibles, souvent guidées par ce qui vient d'être produit, et résultent d'un choix «forcé» (voir Brown 2000). Pour le dire autrement, et au-delà de l'opposition simpliste intuition/réflexion, la créativité musicale ne prend sans doute pas les mêmes formes dans les deux cas même si, là encore, nous aurions bien du mal à identifier des formes de créativité purement compositionnelles ou purement improvisatoires (Clarke 2011, Bertinetto 2011, Dietrich et Kanso 2010).

Dans cette perspective, il peut être particulièrement intéressant, pour faire ressortir la spécificité de ces deux facettes de la créativité musicale, d'examiner la manière dont elles peuvent s'articuler au sein d'un processus de création donné, et de chercher à comprendre quels peuvent être leurs rôles respectifs privilégiés. En particulier, si l'on identifie souvent la dimension compositionnelle des processus d'improvisation aux matériaux dont hérite l'improvisateur (modèles, référents, schémas, éléments stylistiques patiemment 
incorporés par le musicien...) et qui sont ensuite travaillés, ré-élaborés et modifiés par lui dans le temps de l'improvisation, il faudrait également pouvoir identifier et caractériser la dimension improvisatoire de certains processus compositionnels, ou du moins tenter de comprendre le rôle joué par l'improvisation-et la forme de créativité qui lui est associée-dans ces processus. Or, la tâche semble ici beaucoup plus délicate: en effet, outre les traditionnels problèmes soulevés par toute tentative de reconstitution génétique a posteriori-encore que les chercheurs semblent rivaliser d'ingéniosité depuis quelques temps pour surmonter ces problèmes (voir Donin 2012 pour une synthèse)_il semble particulièrement difficile de parvenir à percer l'intériorité du compositeur pour dévoiler ce qui, in fine, dans le secret de son atelier, relève tantôt d'une facette de la créativité musicale, et tantôt de l'autre. ${ }^{5}$ Comme le rappelle William Kinderman: «opposer l'improvisation à la composition à proprement parler ne permet pas de comprendre correctement le processus créatif [de Beethoven], car c'est ignorer l'interdépendance cruciale qui peut exister entre liberté et détermination dans le contexte stylistique concret dans lequel Beethoven a travaillé» (Kinderman 3); et cette remarque sur l'interdépendance de l'improvisation et du travail compositionnel chez Beethoven vaudrait sans doute tout autant pour d'autres compositeurs qui entretiennent une relation particulière à l'improvisation, comme Chopin, Schumann, Debussy, Scelsi ou encore Dusapin.

Cet article se propose précisément d'étudier la manière dont composition et improvisation peuvent se trouver articulées au sein d'un processus de création musicale, et plus particulièrement la place que peut occuper l'improvisation dans un processus compositionnel. Pour cela, nous analyserons le processus de création d'une œuvre pour clarinette et piano, Fenêtre Ovale (2011)—série de 13 pièces du compositeur français Karl Naëgelen (né en 1979)—dont la spécificité est d'avoir été composée en relation étroite avec un duo d'improvisateurs s'exprimant dans le champ de l'improvisation dite «libre»—la pianiste Ėve Risser et le clarinettiste Joris Rühl. Après avoir indiqué ce qui fait l'intérêt de Fenêtre Ovale pour le problème que nous souhaitons aborder, nous montrerons que l'improvisation a pu occuper trois fonctions essentielles dans le processus de création de cette œuvre: une fonction de stimulation, une fonction d'inspiration et une fonction de simulation. Toutefois, le compositeur de Fenêtre Ovale entend également ériger l'improvisation en paradigme de la composition. À cet égard, le processus de création de l'œuvre peut également se lire comme un processus d'absorption de l'improvisation par la composition, démarche qui n'est pas sans soulever tensions et paradoxes: c'est cette démarche que nous questionnerons pour finir, en indiquant ce qu'elle permet de révéler des spécificités respectives du geste compositionnel et du geste improvisatoire.

\section{Fenêtre Ovale: un laboratoire d'analyse}

Dans la mesure où elle mêle improvisation et composition, il est évidemment possible de rattacher Fenêtre Ovale au champ de la " comprovisation » évoqué ci-dessus. Mais Fenêtre Ovale occupe en réalité une place particulière au sein du continuum de la comprovisation et de ses mille et une manières d'hybrider improvisation et composition. En effet, dans la plupart des collaborations entre compositeur et improvisateur, ce sont typiquement dans les moments de performance que l'on peut observer de manière privilégiée l'interaction entre éléments composés et éléments improvisés. L'improvisation est alors bien plus du côté du produit crée que du processus de création: s'il y a bien une distribution de la créativité entre le compositeur et les musiciens-improvisateurs, celle-ci se situe plutôt en aval de l'œuvre-lors des phases de répétition ou des moments de concert—qu'en amont-lors de la gestation du matériau musical, ou de la forme de l'œuvre. ${ }^{6}$

Or, dans le cas de Fenêtre Ovale, la place de l'improvisation et des improvisateurs est entièrement sousjacente à l'œuvre. Si l'improvisation est, en un sens, au cœur du projet compositionnel, elle n'affleure jamais véritablement à la surface de l'œuvre: la partition ne comporte aucune incitation explicite à l'improvisation et, même si elle été composée pour être interprétée au premier chef par les deux musiciens déjà mentionnés, le compositeur envisage tout à fait que son œuvre puisse être jouée par un duo de musiciens n'ayant pas particulièrement de pratique de l'improvisation. Autrement dit, c'est bien dans le processus de création luimême, plus que dans le produit de cette création, que l'on peut observer l'articulation entre composition et improvisation, ce qui est évidemment essentiel pour notre propos.

Fenêtre Ovale se distingue également des innombrables œuvres de musique contemporaine qui sont conçues en étroite relation avec des interprètes particuliers, et qui peuvent accompagner le processus de création en suggérant au compositeur des modes de jeu, en vérifiant avec lui la jouabilité ou l'idiomaticité de tel ou tel passage ou encore en proposant des solutions à certaines questions de notation. ${ }^{7}$ Car si cet aspect est évidemment bien présent dans Fenêtre Ovale, ce n'est pas au premier chef en tant qu'interprètes que les musiciens impliqués ont participé à l'élaboration de l'œuvre, mais bien en tant qu'improvisateurs, c'est-à-dire, d'une certaine manière, en tant que créateurs concurrents de musique-même si, et nous y reviendrons, ils deviennent au final interprètes de cette œuvre.

L'étude du processus de création de Fenêtre Ovale devrait donc nous permettre de mieux comprendre la place que peut occuper l'improvisation dans un processus compositionnel. Pour mettre au jour ce processus de création dans toute sa complexité, nous nous sommes appuyés sur de multiples données:

1. Une série d'entretiens exploratoires avec le compositeur et les improvisateurs 
2. Les esquisses de l'œuvre, rassemblées par le compositeur, ainsi que les enregistrements audio réalisés par le compositeur ou par les improvisateurs à l'occasion du travail sur Fenêtre Ovale (septembre 2009janvier 2011)

3. Une série de séances de verbalisation avec le compositeur et avec les improvisateurs, tantôt à partir des esquisses, tantôt à partir des enregistrements, visant à clarifier ou à expliciter leurs rôles et leurs places dans le processus de création

\section{Plusieurs enregistrements d'improvisations réalisées par le duo concurremment à l'élaboration de Fenêtre Ovale}

Avant de continuer, il nous faut néanmoins répondre à une question essentielle: est-il juste d'identifier le processus de création de Fenêtre Ovale à un processus essentiellement compositionnel? II serait en effet absurde de remettre en cause la dimension collaborative de ce processus de création, et l'opération de distribution de la créativité que celle-ci implique. De manière générale, l'acte de création n'est pas imputable au seul compositeur, pris dans son splendide isolement, mais s'inscrit au contraire dans un ensemble de médiations musicales, sociales et technologiques (Born 2005); et c'est évidemment encore plus vrai dans le cas de projets comme Fenêtre Ovale, qui sont construits autour d'une collaboration étroite entre compositeurs et instrumentistes (voir Bayley et Clarke 2011).

Il n'y a donc en réalité aucune raison a priori pour privilégier le point de vue du compositeur, et placer le travail du compositeur Karl Naëgelen au cœur du processus de création de Fenêtre Ovale. Les improvisateurs, surtout ceux qui s'expriment dans le champ de l'improvisation libre, sont des êtres «hybrides» puisqu'ils sont, dans le même temps, créateurs-faisant d'ailleurs parfois référence à leur activité comme à une activité de composition à part entière (voir Rose et MacDonald 2012)—et exécutants de leur propre musique. Dans ce genre de collaborations, ils sont donc susceptibles d'occuper différents points sur un continuum, dont les extrêmes seraient, d'un côté, l'assimilation des improvisateurs à des interprètesparticipant à l'élaboration de l'œuvre du strict point de vue de l'instrumentiste-et de l'autre, leur assimilation à des co-compositeurs, participant à l'élaboration de l'œuvre avec le compositeur en titre dans une horizontalisation stricte de la collaboration. La question est donc de savoir comment, dans le cas de Fenêtre Ovale, se sont organisées les relations entre le compositeur et les improvisateurs.

II y a évidemment quelque chose d'atypique dans ce projet de collaboration entre un compositeur et des improvisateurs qui ne se cristallise pas dans une performance semi-improvisée, ce qui a en général le mérite de clarifier la répartition des rôles: typiquement, au compositeur la conception du cadre compositionnel dans sa globalité; aux improvisateurs la prise en charge d'une performance comportant des éléments improvisés. Ici, les rôles de chacun se sont définis progressivement tout au long du projet. ${ }^{8}$

Plusieurs raisons nous invitent en réalité à considérer le processus de création de Fenêtre Ovale comme un processus essentiellement compositionnel avec une forte composante improvisatoire, plutôt que comme un processus «comprovisatoire» dans lequel compositeur et improvisateurs travailleraient à égalité à la réalisation d'un projet musical commun, et ce en dépit des intentions qui ont pu animer les trois protagonistes dans le montage du projet. ${ }^{9}$ De manière générale, il est évident que le mode opératoire qui se met progressivement en place entre les improvisateurs et le compositeur s'inscrit dans un cadre implicite fort puissant, celui de la relation compositeur/interprète, qui contraint et oriente les interactions musicales entre les différents protagonistes:

1. Fenêtre Ovale s'inscrit dans le circuit traditionnel de la composition savante en France (il s'agit d'une commande d'État), qui institue le compositeur, et lui seul, comme moralement et juridiquement responsable de l'œuvre.

2. Le processus de création est tout entier orienté vers la composition d'une partition-partition relativement déterminée, qui plus est, ne laissant guère de place à l'improvisation-plutôt que d'un concert, d'une performance, ou d'un spectacle, donc vers la constitution d'un texte qui, de par le processus de fixation dont il fait l'objet, reste dans une certaine mesure "extérieur» aux improvisateurs, et se voit immédiatement conférer par eux une très nette dimension d'autorité. ${ }^{10}$

3. Ensuite, si l'on examine la manière dont s'est déroulé concrètement le processus de création, il est frappant de constater qu'il y a finalement eu assez peu d'allers-retours entre propositions écrites et réactions improvisées. Passé une première étape qui a consisté essentiellement en de longues séances d'improvisation du duo, avec le compositeur qui écoutait silencieusement, en prenant quelques notes, le compositeur a commencé à diriger des séances d'enregistrement et d'archivage de sons. ${ }^{11}$ Ce travail a débouché sur une première série de trois miniatures, qui se sont bien sûr modifiées jusqu'à la création, mais finalement assez peu: en examinant le dossier génétique, on se rend compte que les ajustements et modifications que le compositeur apporte à sa partition suite à une interaction avec les musiciens vont quasisystématiquement dans le sens de davantage de précision, de détermination, et non dans le sens d'une 
modification substantielle, qui serait opérée en réaction à une proposition des musiciens. ${ }^{12}$ Pour le dire autrement, une fois le travail du compositeur bien enclenché, les musiciens ont certes toujours été associés au processus de création, mais peut-être davantage comme interprètes que comme improvisateurs. ${ }^{13}$ II y a donc eu deux étapes assez distinctes dans le processus de création: l'une caractérisée par la passivité du compositeur, en situation de retrait; l'autre caractérisée au contraire par la reprise en main du compositeur, qui amène aux musiciens des propositions écrites plus ou moins finalisées et qui dirige la plupart des séances de travail.

4. Enfin, on peut noter qu'il n'y a au final guère de questions quant à la paternité effective de l'œuvre:

Je me suis dit à la fin de l'enregistrement, quand on a concrétisé le projet... j'étais un peu frustré, et on est passé dans la régie pour tout écouter. En fait j'étais content de la musique et je me suis dit: «c'est de la chouette musique, mais c'est celle de Karl, il n'y a pas de doutes». (Joris Rühl [JR], entretien du 17/09/2012)

S'il avait refait notre musique, ça aurait été moins intéressant: en tout cas, moi, ça ne m'intéresse pas de rejouer ce que je fais, écrit [. . . ]. Je sens [des choses] du duo et je sens des choses que je ne connais pas, mais ça reste la musique de Karl [. . . ]. J'ai l'impression que je vois vraiment un trio, et en même temps je n'ai aucun problème à reconnaître le compositeur et son autorité. (Ėve Risser [ER], entretien du 13/10/2012)

Je pense que c'est de la musique que j'ai composée, et qui n'aurait pas pu être composée autrement qu'à travers ce projet collectif. Mais ça m'a rassuré, quand même, parce que même Ėve et Joris, la première chose qu'ils m'ont dite, c'est: «c'est ta musique.» Le retour que j'ai eu, aussi, c'est que les gestes instrumentaux étaient utilisés par des chemins qu'ils n'auraient pas imaginés, eux. Ce n'était pas leur chemin. (Karl Naëgelen [KN], entretien du 26/09/2012)

Pour toutes ces raisons, nous pouvons considérer le processus de création de Fenêtre Ovale comme un laboratoire d'analyse pertinent pour questionner les places, rôles et fonctions de l'improvisation dans le processus compositionnel lui-même. Dès lors, la dimension collaborative du processus de création, loin d'obscurcir l'analyse, peut au contraire se transformer en un atout considérable dans la perspective qui est la nôtre: le fait que les différentes modalités poïétiques (composition/improvisation) soient assignées à des personnes physiquement distinctes (le compositeur d'un côté, les improvisateurs-interprètes de l'autre), en interaction soutenue les uns avec les autres, permet aussi d'extérioriser, de manifester explicitement le rôle qui peut être joué par l'improvisation dans un processus compositionnel, et de mieux comprendre l'articulation qui peut exister entre ces deux modalités de création.

\section{Rôles et fonctions de l'improvisation dans le processus compositionnel: stimulation, inspiration et simulation}

Il ressort de l'analyse du corpus présenté ci-dessus que l'improvisation a joué essentiellement trois rôles dans le processus de composition de Fenêtre Ovale:

1. Un rôle de stimulation

2. Un rôle d'inspiration

3. Un rôle de simulation

Ce sont ces trois fonctions de l'improvisation que nous détaillons ci-dessous.

A. Improvisation et stimulation du processus compositionnel

L'improvisation revêt d'abord un rôle de stimulation: elle met en branle l'imagination (et l'imaginaire) du compositeur en faisant émerger de nouvelles idées ou envies compositionnelles. Cette fonction de stimulation est non seulement chronologiquement première dans le processus de création de Fenêtre Ovale, mais également logiquement première, dans la mesure où le projet compositionnel même de Fenêtre Ovale n'existe que dans son articulation aux propositions improvisées du duo Risser/Rühl:

Il y a eu un gros moment d'écoute; j'ai passé un temps fou à les écouter. Par rapport à un projet lambda (commande d'un ensemble, par exemple), le temps qu'on a passé ensemble a peut-être été multiplié par dix ou quinze, ce qui est un luxe qu'on ne se permet pas forcément. Donc j'ai passé beaucoup de temps à les écouter improviser, sachant que j'ai rarement été autant stimulé par eux que quand ils improvisaient. Ils pouvaient me montrer leurs sons après, à froid, ce n'était pas pareil. C'était quelque chose de fastidieux, et pas du tout moteur; alors que les entendre improviser, 
c'était... j'entendais plein de matières et puis tout à coup je me projetais telle matière dans autre chose qui pourrait devenir... Ou un petit moment, et puis je me disais: ça, développé, ça pourrait faire une pièce. Donc, là, beaucoup de désirs en écoutant toutes ces matières. (KN, entretien du 26/09/2012)

Cette fonction de stimulation dérive essentiellement du lien étroit existant entre improvisation (notamment les formes d'improvisation dites «libres») et exploration instrumentale. L'improvisateur crée de la musique à son instrument, avec son instrument, par son instrument, pour son instrument, parfois même contre son instrument... et l'on pourrait sans doute prolonger cette liste énumérant les différents rapports prépositionnels qui unissent l'improvisateur à son instrument. II semble donc absurde de vouloir considérer l'improvisateur abstraction faite de son instrument. La créativité de l'improvisateur se pense dans le rapport à l'instrument: cela ne signifie aucunement que cette créativité soit de nature purement instrumentale, ou technique; cela signifie simplement que l'instrument est le lieu privilégié où se cristallise la créativité et l'inventivité de l'improvisateur. «L'instrument, voilà ce qui compte: c'est le matériau, la préoccupation véritable» rappelle justement Steve Lacy (cité dans Bailey 110). Si l'on ajoute à cela le contexte musical général dans lequel la pratique de l'improvisation libre s'est développée-l'émancipation du timbre comme paramètre autonome du discours musical, contribuant à faire de l'inouï une des valeurs constitutives de la musique contemporaine de ce dernier demi-siècle-et l'horizon «non-idiomatique» dans lequel s'inscrit cette pratique-qui conduit les improvisateurs à éviter de centrer leur discours sur la manipulation des paramètres qui sont justement, dans la mémoire collective de ces musiciens, les paramètres les plus fortement chargés de prédétermination idiomatiques (le couple hauteurs/durées) ${ }^{14}$-il n'est guère étonnant que l'extension et la singularisation (voir Walter 2006) du timbre instrumental constitue un des objectifs régulateurs de la pratique de l'improvisation libre.

Les musiciens envisagent alors l'improvisation libre comme une sorte de laboratoire leur permettant d'explorer, puis de développer un rapport à l'instrument et, par delà l'instrument, au sonore, qui leur soit spécifique. Le monde de l'improvisation libre peut être vu comme une galaxie de rapports extrêmement singuliers à l'instrument; ces rapports naissent bien sûr des altérations possibles que les musiciens font subir à l'instrument (notamment la préparation de l'instrument), des augmentations diverses et variées qu'ils lui adjoignent (élaborations de patchs informatiques, effets électro-acoustiques...) —bref, de tout ce qui permet d'étendre la lutherie traditionnelle-mais aussi d'une recherche spécifique sur les gestes instrumentaux. Ce qui est en jeu, ici, c'est donc bien le savoir de l'improvisateur en tant qu'instrumentiste, certes, mais un instrumentiste un peu particulier, qui fait de l'exploration de son instrument un des centres spécifiques de son activité créative. Les deux musiciens impliqués dans le projet Fenêtre Ovale n'y font bien sûr pas exception, puisqu'ils possèdent un très large vocabulaire instrumental-fruit de leurs recherches d'improvisateurs-qui plus est articulé à un objectif de métamorphose de l'instrument, par un dépassement des limites et contraintes organologiques dont il est porteur. ${ }^{15}$

Le clarinettiste Joris Rühl décrit d'ailleurs ainsi son rapport à l'instrument: «je suis dans une esthétique qui essaye de faire oublier l'instrument et on a vite fait, du coup, de ne plus entendre que l'instrument quand quelque chose nous échappe. Et c'est ça le plus délicat» (JR, entretien du 17/09/2012). C'est cette capacité des deux improvisateurs à faire oublier leurs instruments, cette transformation de l'instrument propice à la recherche d'illusions sonores et acoustiques inédites qui a fortement stimulé le compositeur:

Naïvement je dirais qu'ils m'ont déjà proposé des choses que je n'aurai pas pu imaginer tout seul. Notamment quand on pense clarinette/piano, on se dit que c'est deux instruments très différents, alors que là ils ont la capacité de faire fusionner des timbres. Deux aspects, donc: individuellement, ils avaient des recherches très poussées sur l'instrument; mais en plus comme c'est un duo d'improvisateurs, ils avaient déjà expérimenté des sons qu'ils pouvaient fusionner, faire des alliages de timbre. Donc il y avait en plus cette dimension collective du travail de recherche. (KN, entretien du 26/09/2012)

Cette pâte sonore du duo Risser/Rühl transparaît très clairement dans la séquence vidéo ci-dessous, qui présente le début d'une improvisation enregistrée par le duo pour le label Umlaut Records en 2010 , accompagné d'une transcription. ${ }^{16}$ On y entend une texture faite d'un suraigu de clarinette et d'un son obtenu en frottant un morceau de polystyrène directement sur les cordes du piano, sans qu'il soit toujours très facile d'identifier la source exacte de chacun des sons qui composent cette texture; les timbres instrumentaux y sont tellement fusionnés que l'on n'a guère l'impression d'un duo-surtout si l'on vise par là une quelconque qualité dialogique-mais bien plutôt d'un unique objet sonore aux contours toujours mouvants. 

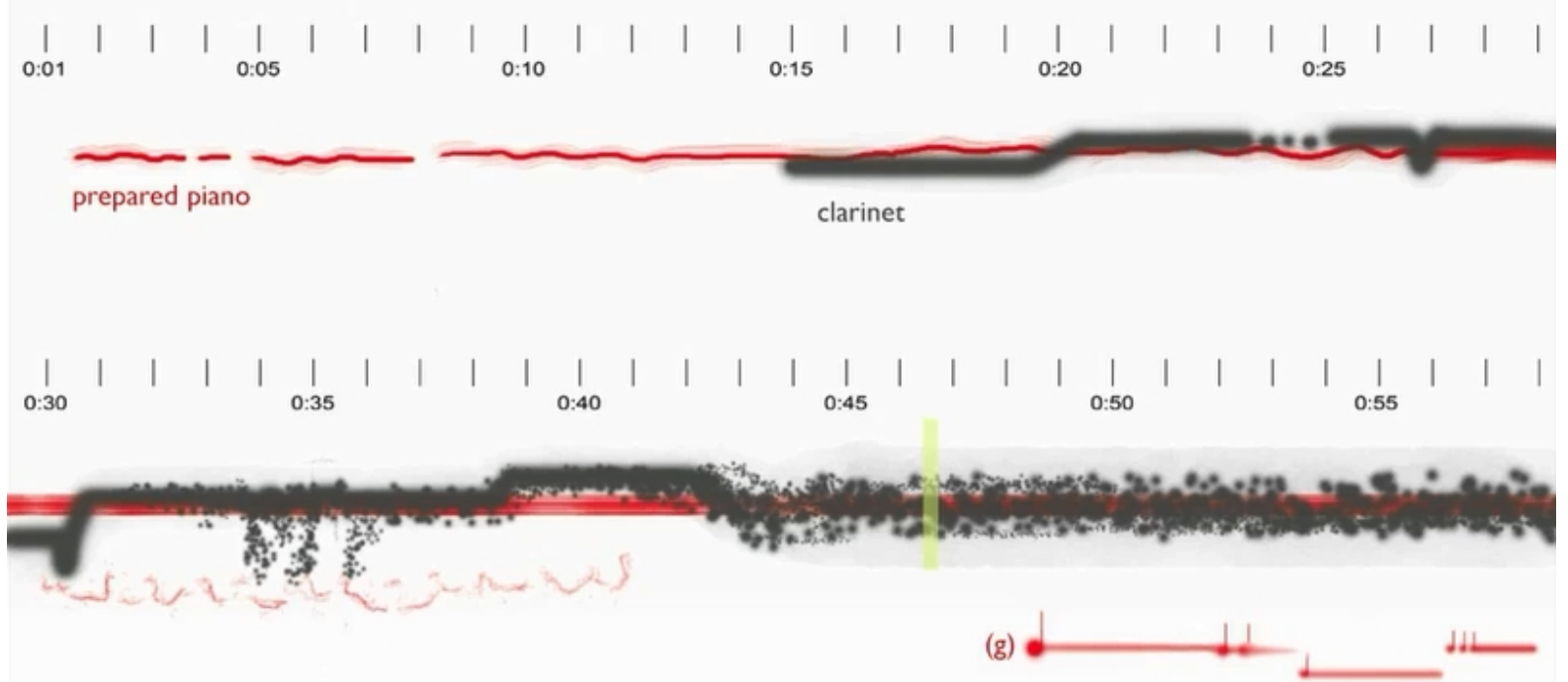

\section{Transcription du début d'une improvisation du duo Risser/Rühl, enregistrée live au festival Piednu, Le Havre, 3 avril 2010.}

Cette fonction de stimulation par l'improvisation ne procède donc pas uniquement des recherches individuelles des deux musiciens, mais également de leurs recherches collectives, qui visent à constituer une esthétique de l'illusion, du trompe l'œil, de la confusion systématique des sources sonores. Cela apparaît nettement dans le travail de catalogage effectué par le compositeur, qui a soigneusement répertorié ce très riche vocabulaire instrumental, à la fois sous une forme écrite et sous la forme d'une sonothèque, où les musiciens présentent leurs diverses techniques de jeu, en explicitant à chaque fois le type de geste requis par cette technique. Ce catalogue ne présente pas une à une les différentes techniques des improvisateurs, dans une tentative d'épuisement de leur répertoire instrumental, mais est plutôt organisé en fonction d'une typologie sonore d'inspiration plus ou moins schaefferienne («souffles,» "percussions harmoniques,» "sons inharmoniques,» «sons itératifs,» "sons continus». . .): ainsi, la première page porte sur les «souffles composites» (c'est-à-dire des sons à hauteur perceptible mais avec une très forte composante bruiteuse), et nous donne à voir différentes techniques permettant d'obtenir ces «souffles composites» aussi bien à la clarinette qu'au piano. On en trouvera une reproduction dans la figure 1 ci-dessous: 


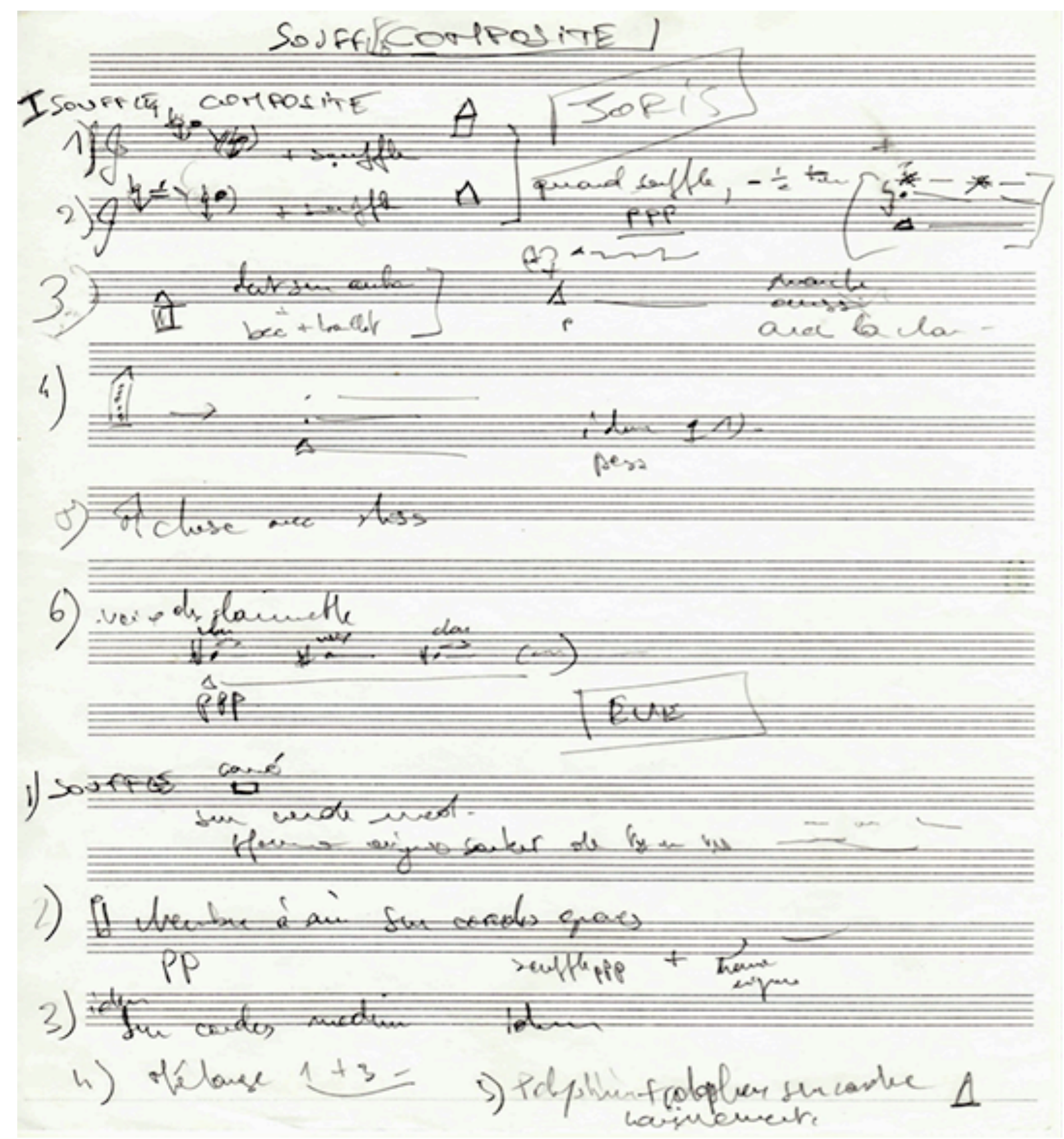

Figure 1. La page «souffle composite» du catalogue de modes de jeu réalisé par le compositeur

Dès cette étape de catalogage, il y a donc l'idée de chercher à fixer comment une même idée sonore peut être réalisée par les deux instruments. Cette volonté d'associer des sons qui se confondent possiblement a clairement structuré le travail du compositeur dans ce projet. ${ }^{17}$ On peut ainsi voir dans la figure 2 comment, suite aux improvisations du duo et parallèlement à ce premier travail de catalogage, le compositeur note, parfois sous la forme de pictogramme, quelques-unes de ces possibles associations de timbres, dont certaines donneront effectivement naissance à des pièces. Le travail collectif du timbre-une des spécificités du duo d'improvisation Risser/Rühl—a donc clairement stimulé l'imagination du compositeur, qui a cherché à son tour de nouveaux alliages de timbre, de nouvelles mixtures sonores susceptibles de se prêter à un travail compositionnel. 


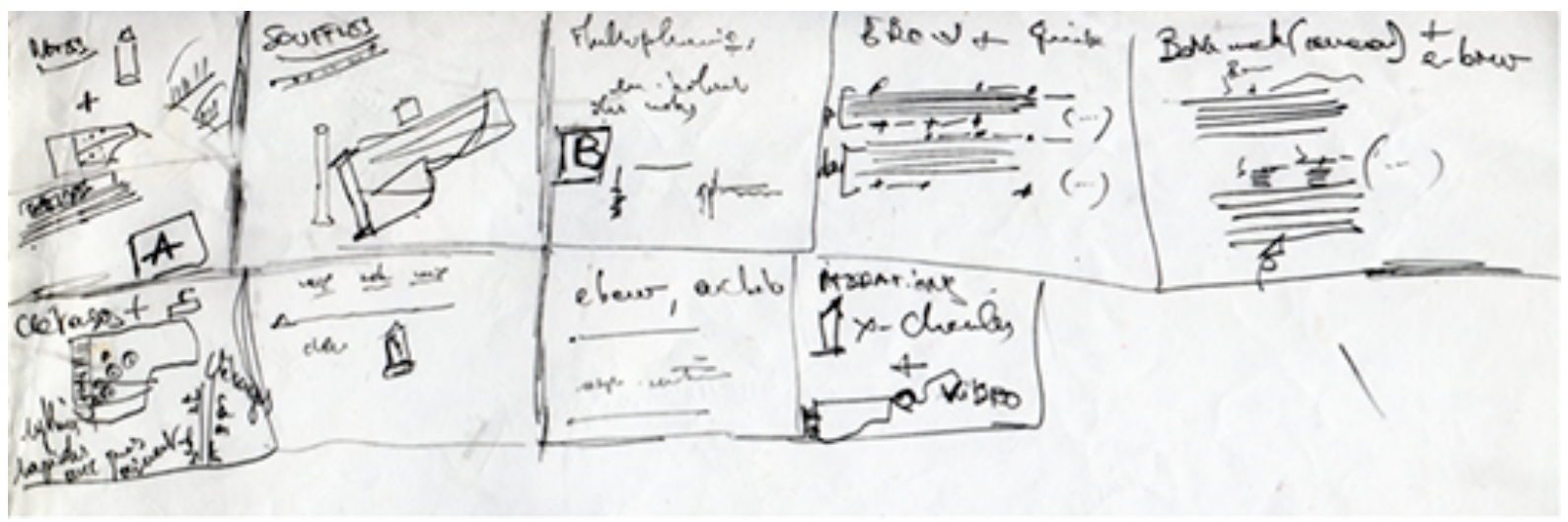

Figure 2. Associations de timbre et/ou de modes de jeu notées par le compositeur

L'influence du paradigme d'improvisation dans lequel se situe le duo Risser/Rühl transparaît donc nettement dans l'importance que revêtent, dans le processus compositionnel, le travail sur le jeu instrumental, les techniques qui lui sont associées, et le timbre résultant. La figure 3 montre qu'en abordant la composition d'une nouvelle pièce (ici «TK vent» qui deviendra «Variations sur Ernst»), le compositeur fixe d'abord une certaine combinaison de timbres et de techniques instrumentales-en l'occurrence des gestes que le compositeur a identifié comme «itératifs» dans son travail de catalogage-avant même d'esquisser les premières ébauches de la partition.

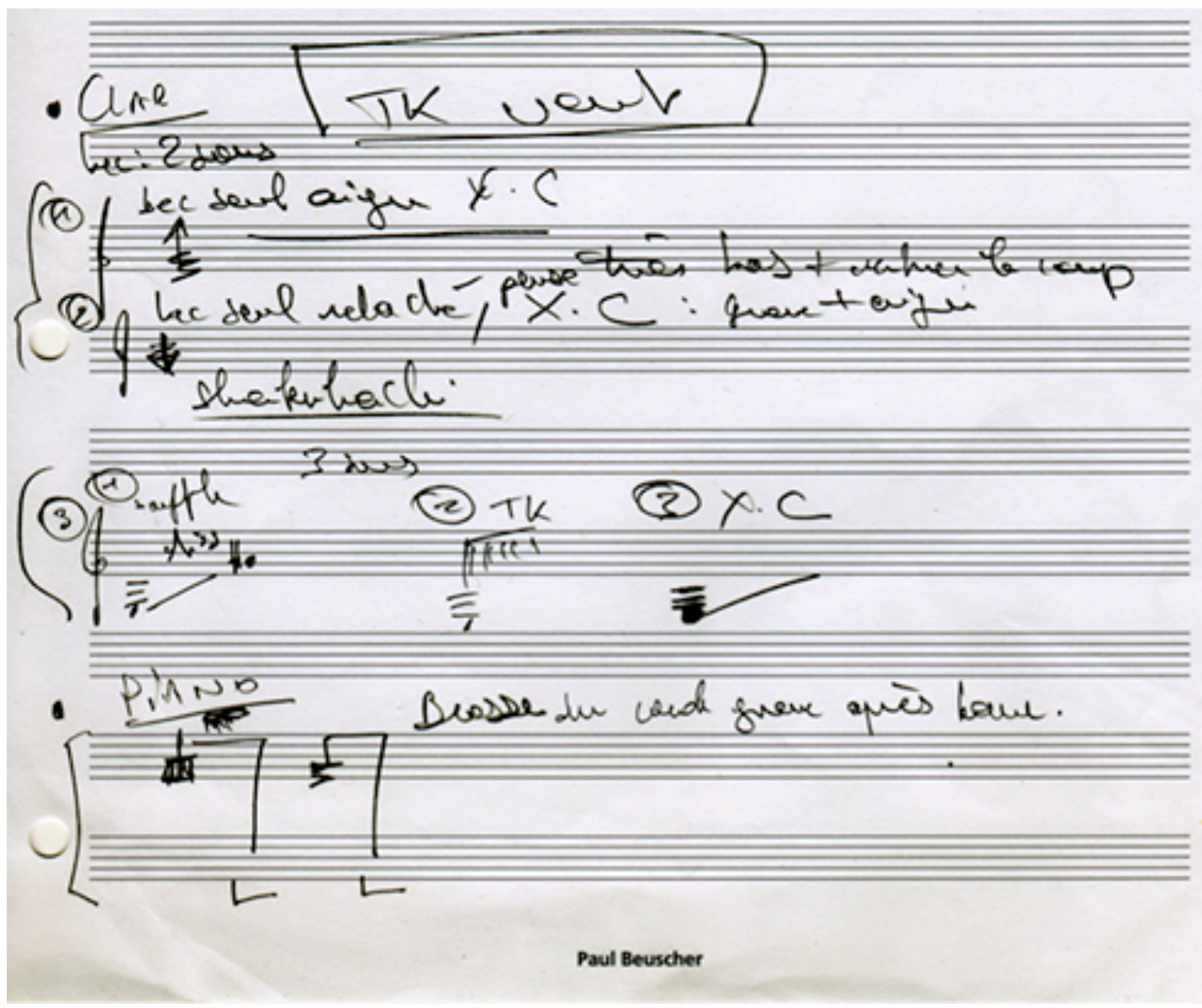

Figure 3. La première esquisse de «Variations sur Ernst»

C'est bien cette dimension de recherche timbrale profondément associée aux musiques improvisées ${ }^{18}$-et le type d'esthétique que l'on peut lui associer-qui apparaît comme motrice dans le processus compositionnel: c'est en tout cas cette fascination pour l'exploration instrumentale si typique de l'improvisation libre qui est à l'origine des envies du compositeur et de ses divers essais de pièces, abouties ou non, tout au long du travail sur Fenêtre Ovale. 19 


\section{B. Improvisation et inspiration}

L'improvisation possède également un rôle évident d'inspiration dans le processus compositionnel de Fenêtre Ovale. Si la fonction de stimulation peut être caractérisée en termes dispositionnalistes-l'improvisation disposant le compositeur à composer, et donc jouant un rôle de déclencheur dans le processus compositionnel-la fonction d'inspiration, elle, se traduit par un transfert effectif, bien que plus ou moins direct, d'idées ou de matériaux musicaux issus de moments d'improvisation vers la composition. ${ }^{20}$

II faut d'abord bien voir que ce vocabulaire instrumental riche et varié dont nous venons de parler est en luimême porteur d'idées compositionnelles, au-delà du seul aspect timbral. Les gestes instrumentaux donnés par les improvisateurs, et que le compositeur entreprend de fixer, sont déjà porteurs, en germe, de formes et de discours musicaux potentiels. II ne s'agit pas d'une matière brute, inerte, mais bien d'une matière déjà préformée, indissociable d'une certaine énonciation temporelle. Le son de l'improvisateur, et le geste qui en est à l'origine, ne sont jamais abstraits d'une certaine temporalisation: ils prennent sens parce qu'ils s'articulent à une certaine situation performative, conformément au lien indissociable qui unit invention et exécution dans le cas de l'improvisation.

Nous pouvons donner un exemple qui illustre bien ce passage de la saisie par le compositeur de ce qui est a priori un pur élément technique du vocabulaire de l'improvisateur à l'idée d'une pièce et à ses phrasés caractéristiques. Dans l'extrait sonore 1, nous entendons le clarinettiste exposer au compositeur un de ses modes de jeu-en l'occurrence en utilisant une balle pour boucher partiellement le pavillon et en soufflant directement dans le corps du haut (sans passer par le bec): s'ensuit une présentation qui prend déjà la forme d'une phrase musicale, utilisant différents sons bruiteux obtenus à l'aide de cette technique. L'extrait sonore 2 permet d'entendre le début de la pièce «Rondo,» qui utilise, entre autres, ce mode de jeu pour la clarinette: on remarque immédiatement la similarité de la conduite de phrasé avec l'extrait précédent. Les différentes matières sonores présentées par les improvisateurs ne font pas que stimuler le compositeur; elles fonctionnent également clairement comme une source d'inspiration, au sens où se trouvent remobilisés dans la composition les modes de temporalisation qui leur sont associés par les improvisateurs.

Cette fonction d'inspiration agit donc jusque dans les détails du processus d'organisation musicale. Mais on peut également observer des phénomènes de transfert beaucoup plus direct et plus évident, dans lesquels c'est une improvisation réalisée par les musiciens qui inspire très nettement l'atmosphère générale, la matière d'ensemble, ou le déroulé global d'une des pièces de Fenêtre Ovale. Nous pouvons en donner deux exemples. L'extrait sonore 3 donne à entendre un moment d'une improvisation réalisée en concert par le duo en novembre 2009, au tout début du travail sur Fenêtre Ovale; le procédé de transformation du timbre à partir d'un son répété rapidement au piano se retrouve très clairement-même si soumis à un processus de condensation—au début de la pièce «Tk» (voir figure 4), et en fournit même le principe moteur, en demandant à la pianiste d'étouffer puis de libérer progressivement les cordes correspondant à la note qu'elle répète $(l a)$. L'extrait sonore 4 fait quant à lui entendre un solo de clarinette qui émerge d'une improvisation réalisée au cours de l'une des séances de travail finales sur Fenêtre Ovale, solo construit autour de l'idée d'un discours continu jouant avec de nombreux quarts de ton. À ce moment, le compositeur avait déjà esquissé l'introduction d'une pièce intitulée «Ombak,» faisant fusionner un trille de clarinette très léger en bisbigliando avec une tenue réalisée à l'aide d'un e-bow placé directement sur les cordes du piano (voir figure 5). Finalement, l'improvisation réalisée ce jour-là inspire très directement le compositeur pour la suite du travail sur cette pièce, en venant s'hybrider avec cette idée initiale déjà notée, pour déboucher sur la version finale d' "Ombak» une pièce pour clarinette seule, utilisant un discours continu en quarts de ton qui vient très progressivement «élargir» la tenue inaugurale, développant ainsi, en le difractant, le principe du bisbigliando d'abord esquissé par le compositeur (voir figure 6). 
$\mathrm{Tk}$
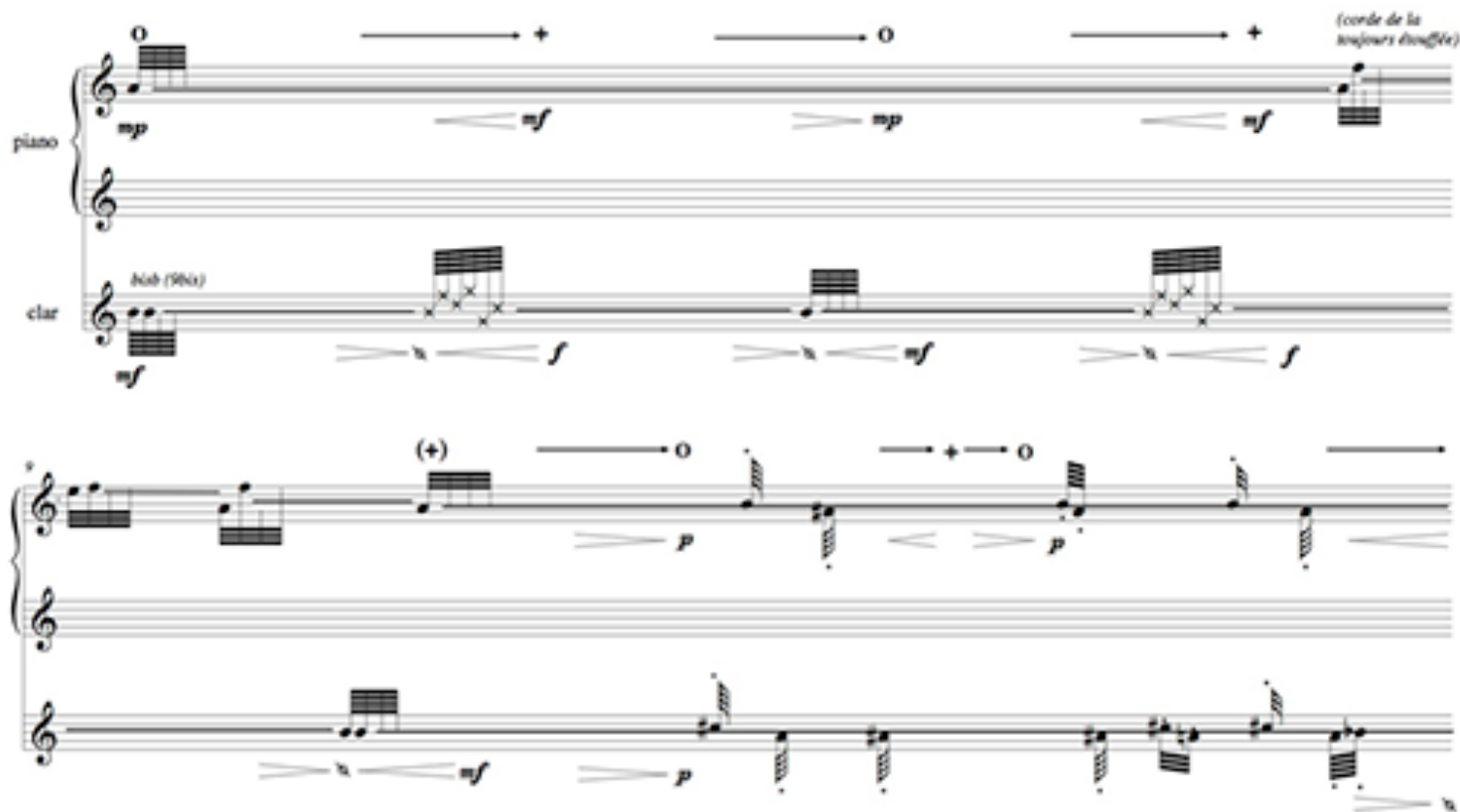

Figure 4. La première page de «Tk»

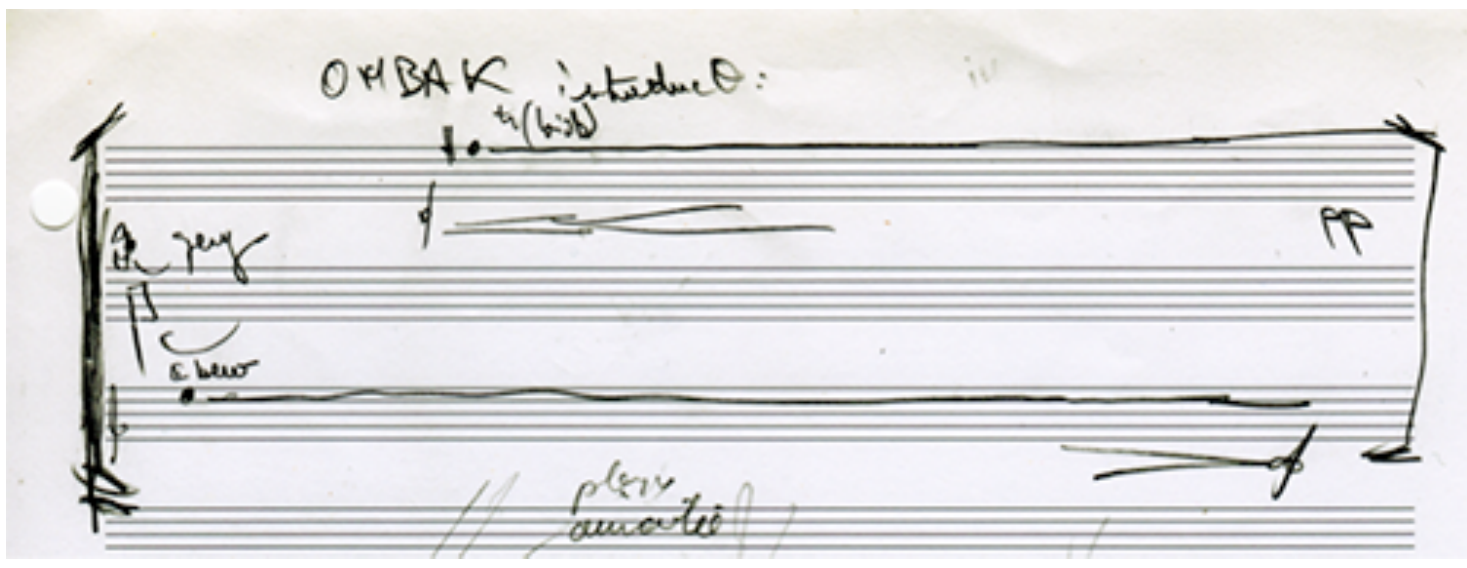

Figure 5. L’idée initiale de «Ombak» 

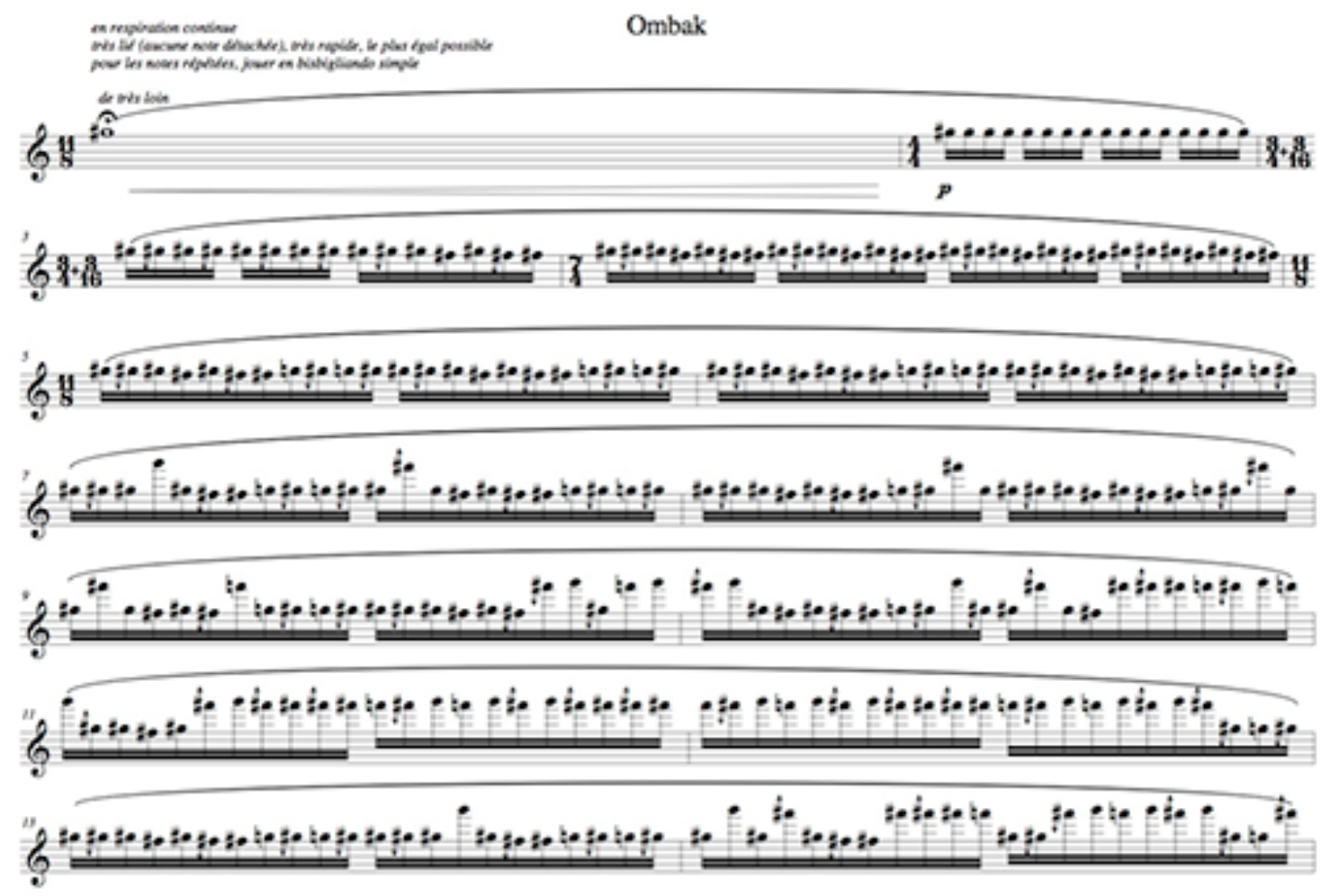

Figure 6. La première page d'«Ombak»

C. Improvisation et simulation

Enfin, l'improvisation joue un rôle tout à fait intéressant dans les phases les plus délibératives du processus compositionnel (voir Pohjannoro 2014): l'improvisation permet en effet au compositeur de simuler différents états de sa composition, en vue de valider, ou au contraire d'invalider, ses choix formels.

Cette fonction intervient à trois niveaux:

1. D'abord, l'improvisation permet au compositeur d'essayer tout de suite une proposition compositionnelle encore incomplète, et d'avoir immédiatement un retour sonore, sensible. II ne s'agit donc pas simplement d'obtenir une idée de la manière dont peut sonner un passage donné en soumettant une esquisse à des interprètes—ce qui est un procédé somme toute assez courant-mais bien plutôt d'envisager l'improvisation comme complétant ce qui est encore à l'état lacunaire. On peut voir dans la figure 7 ci-dessous une esquisse de la pièce «Rondo» qui comporte des parties improvisées, indiquées «Solo» (qu'il faut comprendre ici non pas dans le sens d'un discours en dehors, mais simplement d'un discours librement improvisé) avec une contrainte de timbre (respectivement «pas de sons proches des souffles» pour le piano et «pas de sons éoliens» pour la clarinette). II s'agit donc précisément de tester deux choses: d'abord, la superposition d'un discours très rythmique, assez carré, et d'un discours opérant d'une manière largement indépendante de celui-ci; ensuite la cohabitation entre des matières instrumentales hétérogènes (ici des timbres proches de sons de souffle mélangés à des timbres non-spécifiés, mais explicitement différents des premiers). ${ }^{21}$ Ces parties improvisées ne servent qu'à affiner et à valider l'idée de départ du compositeur; elles disparaissent en effet dans la version finale (voir la figure 8). 


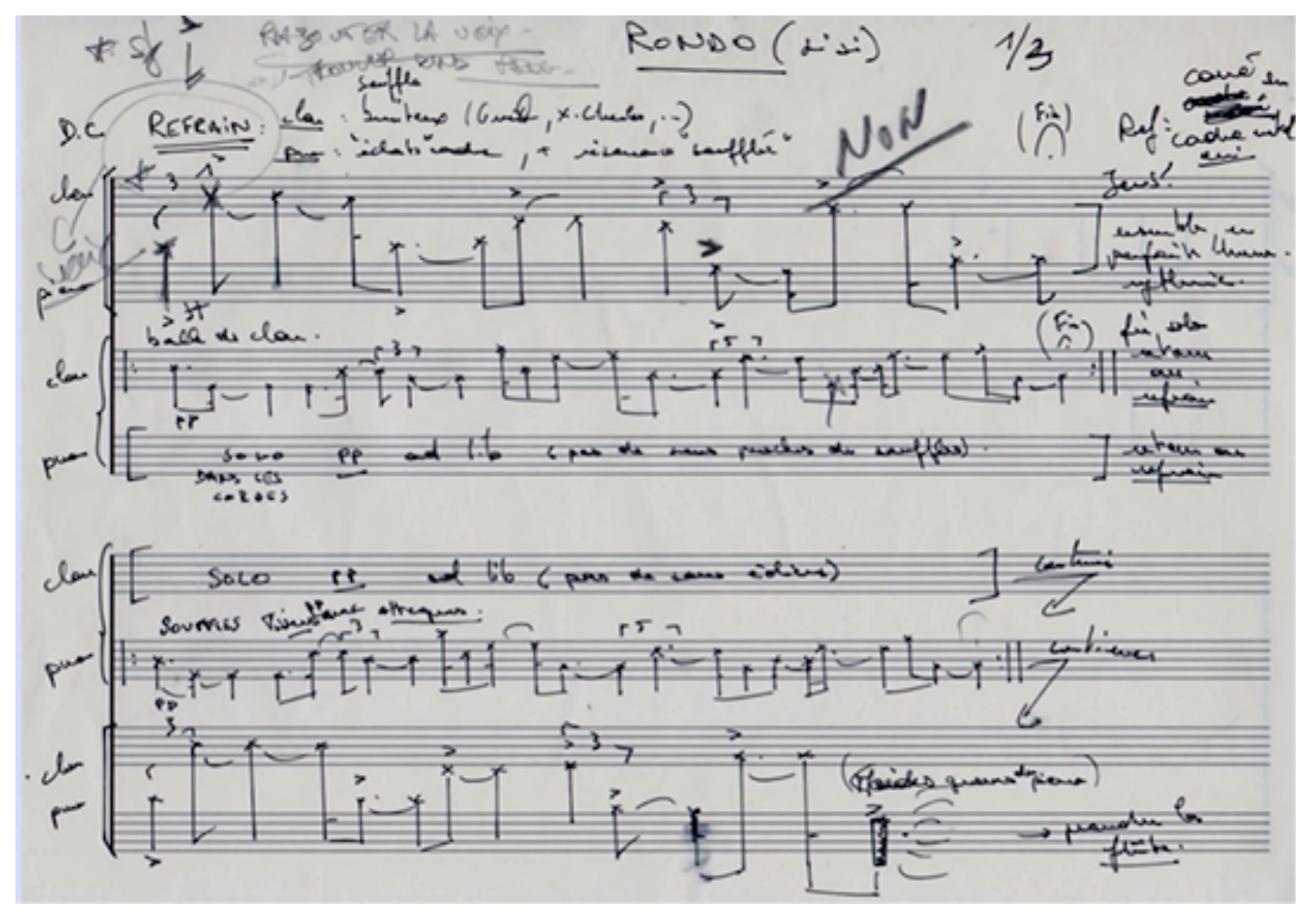

Figure 7. Une des premières esquisses de «Rondo,» avec des parties en «solos» ad lib.

Rondo

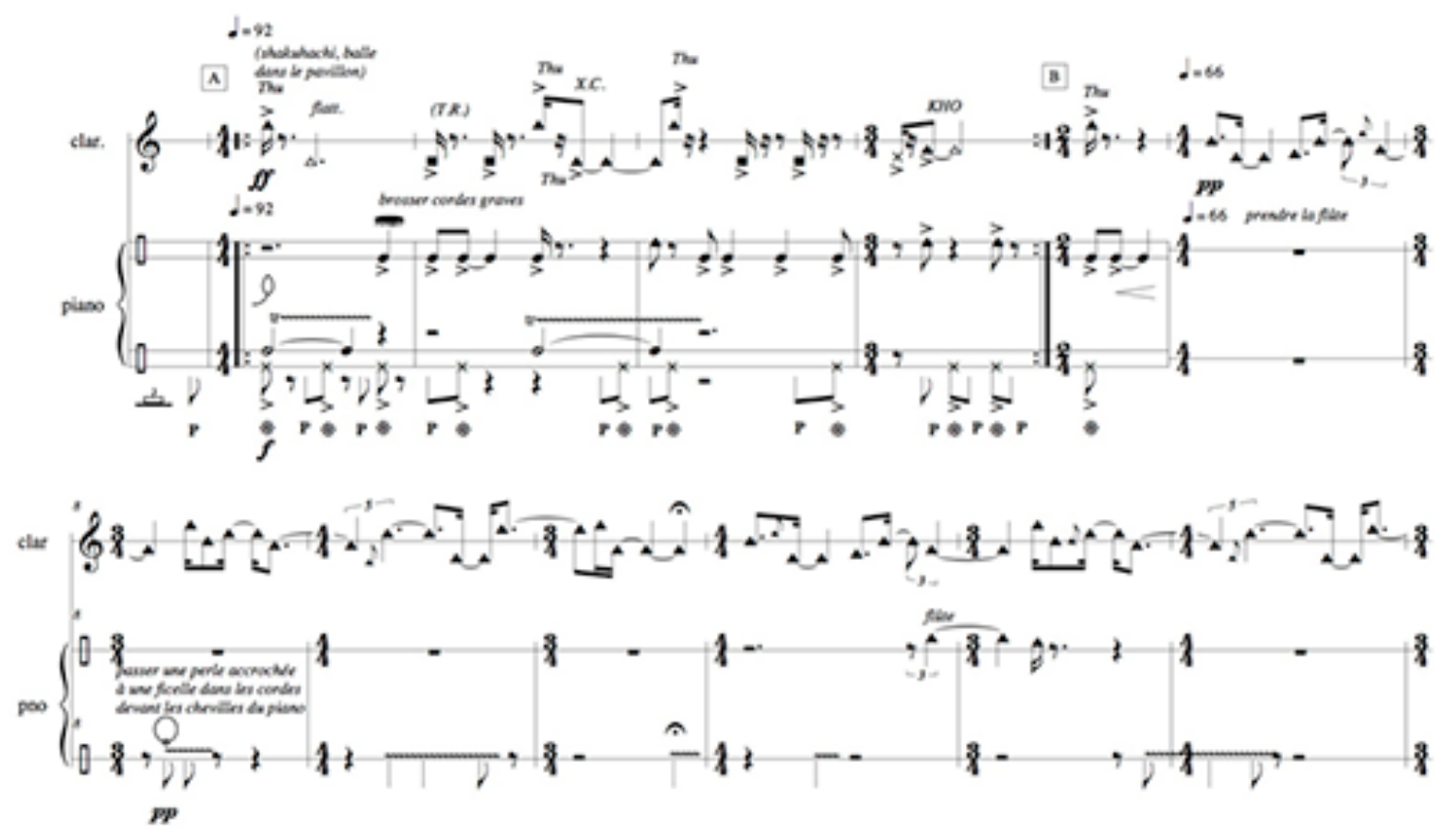


Figure 8. La première page de «Rondo»; les parties improvisées ont disparu des différents «couplets» (lettre B).

2. Ensuite, à une plus large échelle, l'improvisation peut jouer un rôle important pour valider la forme d'une pièce, quand il s'agit de tester l'organisation globale de la pièce ou une possibilité de développement, en examinant par exemple le passage d'un premier matériau à un second matériau assez distinct, que le compositeur désire associer dans la même pièce. C'est typiquement ce genre de processus de validation par l'improvisation qui a abouti à la dissociation de «Kroum» et «Tremuli,» qui ont d'abord été pensées comme une unique pièce. On peut voir dans la figure 9 une esquisse dans laquelle le compositeur fixe le synopsis de ce qui deviendra «Tremuli»; on remarque en particulier dans le deuxième système que le compositeur envisage de passer d'une musique très étale, et fusionnée, à une musique plus animée, avec l'introduction de traits ou, en tout cas, d'un débit perceptible, débouchant sur le passage encadré, indiqué «en impro[visation].» La figure 10 permet de voir un état plus avancé du travail. Dans cette deuxième esquisse, le passage est davantage détaillé: conformément au synopsis précédent, le compositeur ébauche une interpolation pour passer d'une première matière à une seconde matière, obtenue avec une préparation du piano particulière qui vise à se rapprocher des hauteurs que l'on obtient quand on enlève le corps du haut et le barillet de la clarinette (c'est une idée que le compositeur a déjà notée en tant que telle-on peut la lire dans les pictogrammes de la figure 2 ci-dessus_et qui deviendra, in fine, la pièce "Kroum»). On voit que le compositeur semble lutter sur cette transition («changer les notes,» «continuer»), et qu'il hésite entre deux directions formelles possibles, ${ }^{22}$ l'une consistant à «développer,» l'autre à "revenir au son de départ.» Finalement, le passage en question est barré, conformément à ce que l'on trouve dans l'état final de la partition, qui n'utilise pas ce second matériau très contrastant, mais reste dans la couleur initiale.

Mais il est très intéressant de constater que ce renoncement du compositeur n'intervient qu'après avoir demandé aux musiciens, au moins à deux reprises, d'improviser sur un des matériaux envisagés et de passer progressivement, comme par une interpolation, au second matériau. Cette fois-ci, il ne s'agit donc pas d'utiliser l'improvisation pour simuler un tout encore incomplet, mais bel et bien pour simuler dans son intégralité un processus formel—en l'occurrence une transition entre deux «états» musicaux.

Nous avons en effet retrouvé deux improvisations des musiciens qui obéissent au même principe d'organisation, et qui répondent à une contrainte imposée: le compositeur semble avoir demandé aux musiciens d'improviser en partant du matériau plus animé (type «Kroum») pour revenir au matériau très fondu (type «Tremuli») utilisé dans le début de la pièce. L'extrait sonore 5 permet de se faire une idée d'une des improvisations ainsi réalisées. Ces improvisations ont sans doute eu pour fonction de tester la résistance qu'offraient ces matériaux l'un à l'autre et les possibilités de complémentarité qu'ils ouvraient, en cherchant in vivo si l'on pouvait passer "naturellement» ou «spontanément," par fusion ou transformation progressives, de l'un à l'autre. Le compositeur a finalement jugé le résultat suffisamment peu concluant pour abandonner son idée initiale, ou en tout cas la modifier substantiellement: s'il y a bien, au final, une animation progressive de la matière dans «Tremuli,» elle se fait par quelques arpèges de piano qui, assez progressivement, déroulent et complètent l'harmonie sous-jacente au multiphonique de clarinette inaugural (voir la figure 11). C'est donc bien sur la base de ces improvisations/simulations que le compositeur a jugé les matériaux trop disparates et a modifié le dessein formel initialement envisagé pour cette pièce. ${ }^{23}$ 


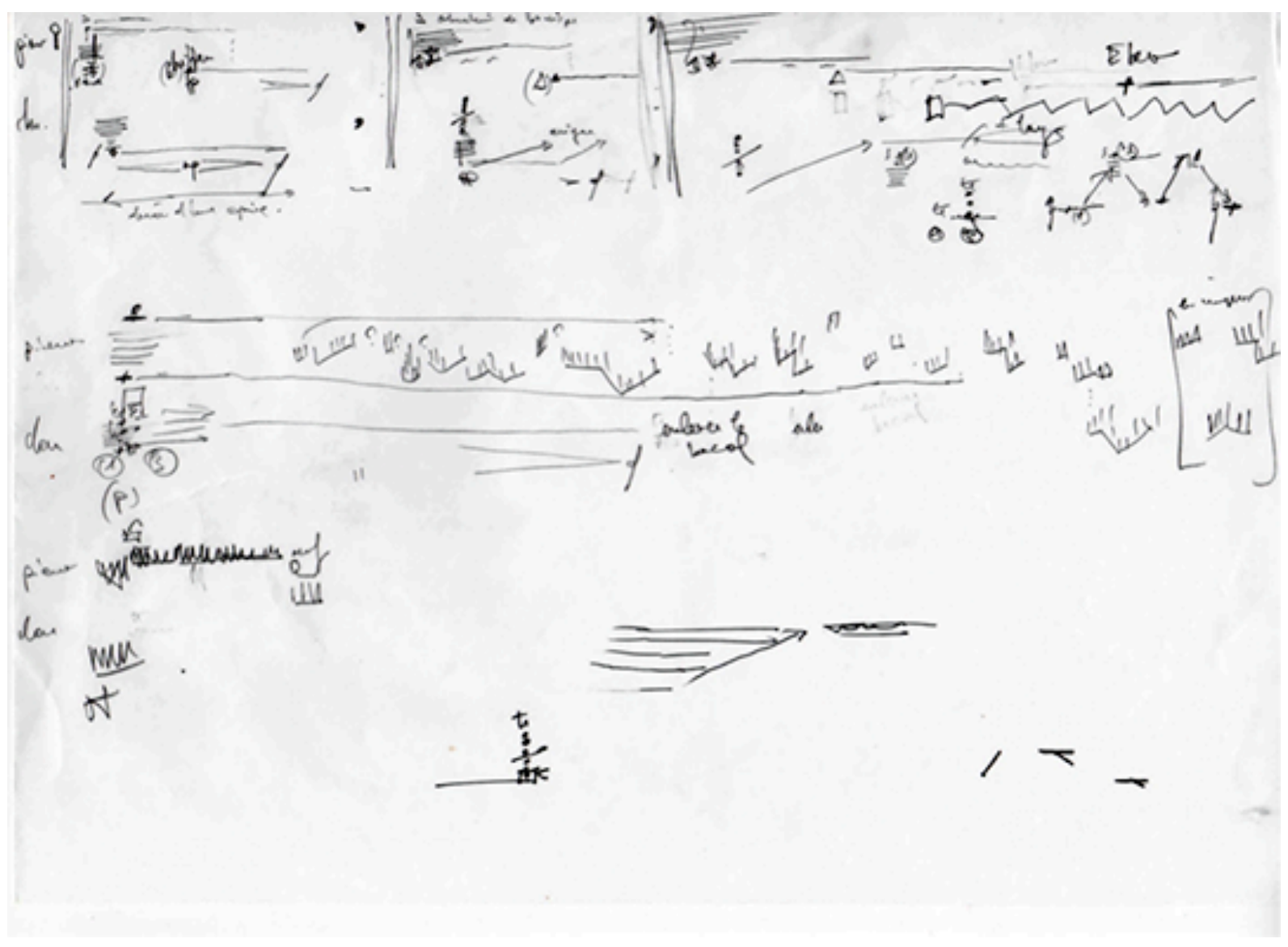

Figure 9. Le synopsis initial de «Tremuli» 


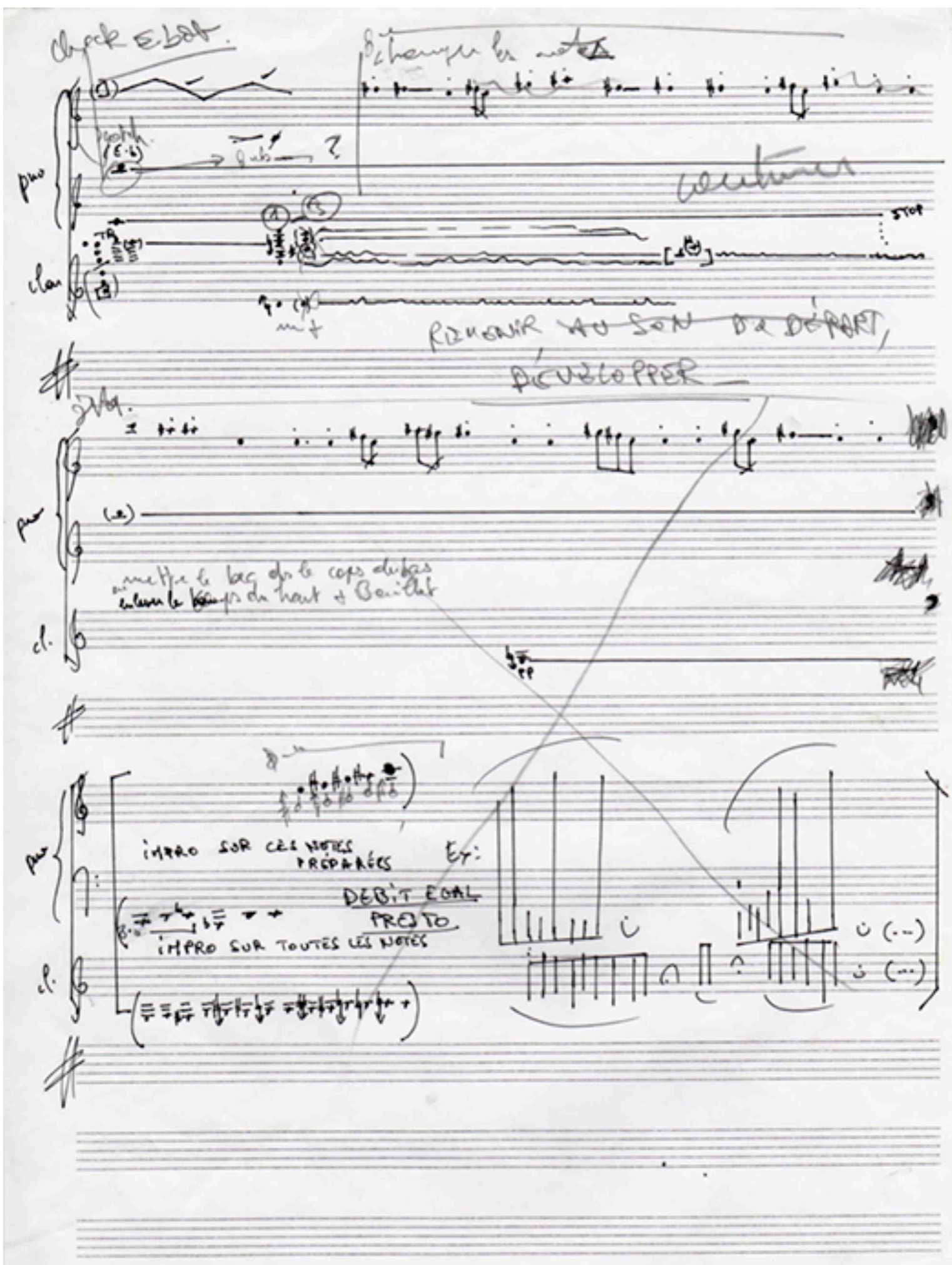

(2)

Figure 10. Une esquisse du développement initialement envisagé pour «Tremuli» 


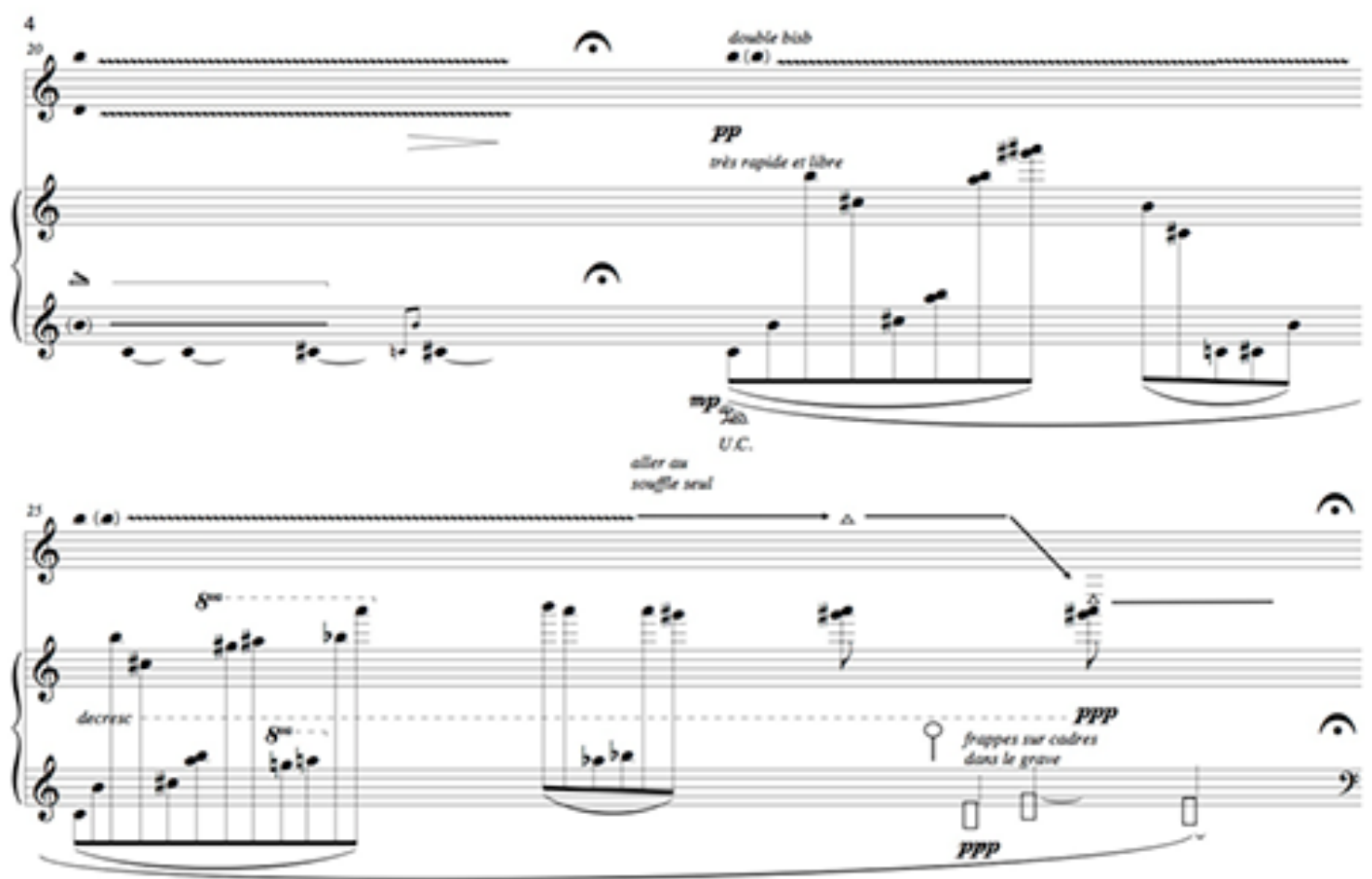

Figure 11. Le développement finalement retenu pour «Tremuli»

3. On peut enfin faire remarquer que l'improvisation a également eu ce rôle de simulation-en complétant ce qui était encore lacunaire-mais à l'échelle de l'œuvre elle-même, quand il s'est agi de mettre en temps les différentes pièces (pour la plupart des miniatures), de les imaginer dans une grande forme, de voir comment elles pourraient s'enchaîner les unes aux autres, se répondre et s'articuler. On voit dans la figure 12, qui provient des étapes plus tardives du processus de création, que l'improvisation a pu être utilisée en ce sens, pour simuler la composition dans son déploiement temporel complet, en intercalant des moments d'improvisation entre les différentes pièces déjà composées, comme pour simuler le rôle que pourraient occuper dans l'équilibre global les pièces encore non-écrites. Cette utilisation de l'improvisation pour compléter le parcours de l'œuvre a notamment permis au compositeur, en testant le déploiement des équilibres musicaux au sein de sa composition, de modifier l'ordre des pièces déjà existantes, et a bien évidemment eu une influence importante sur la manière d'envisager la composition des dernières pièces (comme en témoigne la pièce «Ombak» déjà mentionnée ci-dessus). 


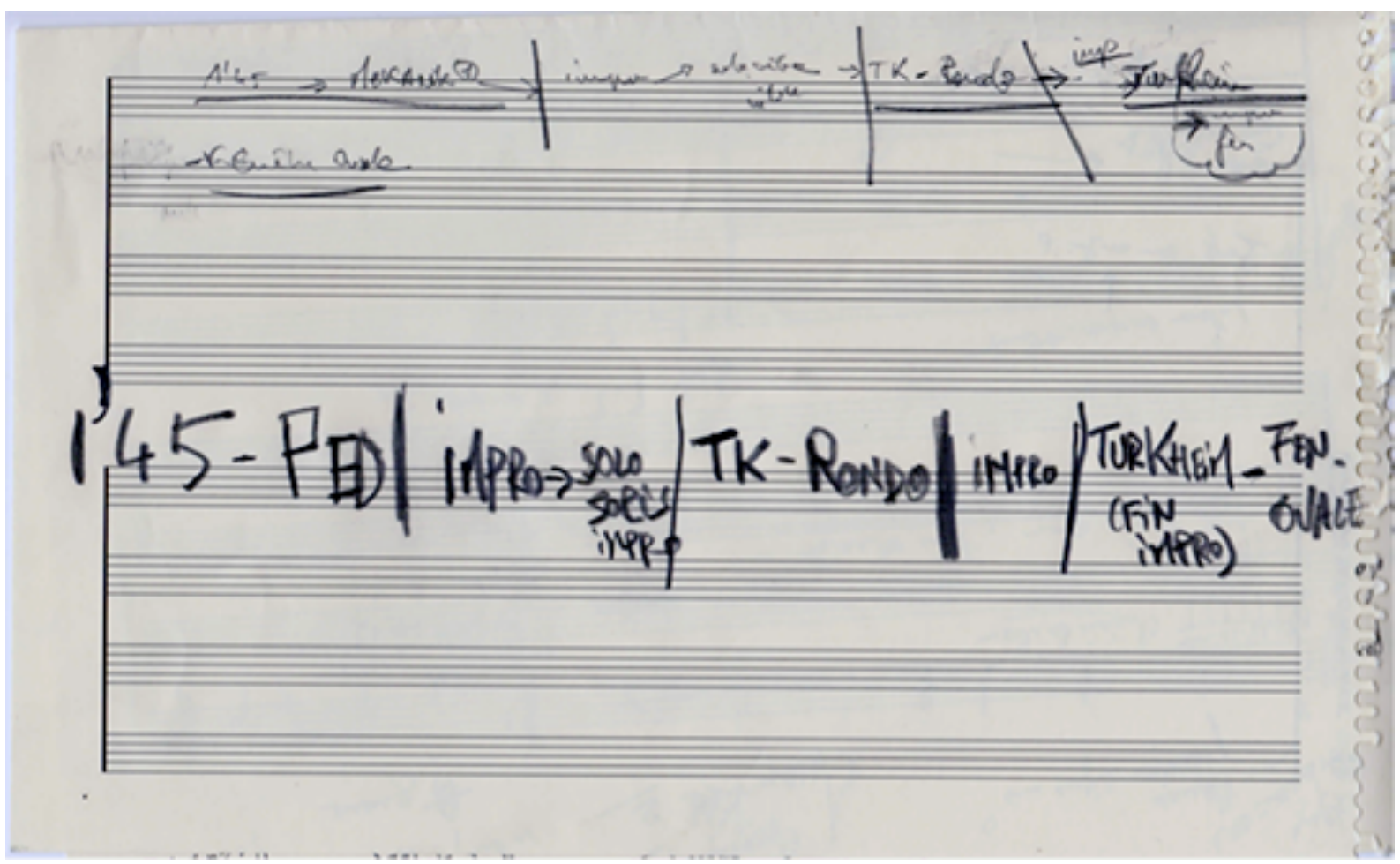

Figure 12. « Déroulé » réalisé par le compositeur demandant aux musiciens d'intercaler des moments d'improvisation entre les pièces déjà écrites.

De cette analyse du processus de création de Fenêtre Ovale se dégagent donc trois fonctions compositionnelles de l'improvisation. Chercher à prêter une portée générale à cette tripartition stimulation/inspiration/simulation nécessiterait évidemment de la confronter à de multiples autres cas. Mais il nous semble néanmoins qu'elle fournit, en première approximation, un point de départ crédible pour aborder et étudier plus avant les diverses places que peut occuper l'improvisation dans le processus compositionnel.

\section{L'improvisation comme horizon de la composition: un processus d'absorption?}

Toutefois, on manquerait peut-être l'essentiel de Fenêtre Ovale en ne considérant l'improvisation que comme une sorte d'auxiliaire ou d'adjuvant de la composition. Il est bien évident que l'improvisation anime le projet compositionnel de Karl Naëgelen de manière encore bien plus profonde; à bien des égards, celui-ci cherche en effet à capturer certaines des propriétés les plus caractéristiques de l'improvisation, et à ainsi créer l'illusion d'une musique qui s'invente dans le temps de sa performance:

Le compositeur qui veut travailler avec des improvisateurs, c'est possible qu'il veuille le beurre et l'argent du beurre. Je me situe sur un endroit un peu risqué; ça reste un projet très écrit, mais ça aurait un peu la prétention de garder une part de la spontanéité de l'improvisation. (KN, entretien du 26/09/2012)

Cela pose évidemment une question essentielle: cette absorption de l'improvisation par la composition ne correspond-elle pas à une dépossession des improvisateurs? Cette crainte est très présente chez la pianiste, qui a déjà connu des expériences peu satisfaisantes avec d'autres compositeurs: «ll y a pas mal de compositeurs qui te prennent des sons et qui font une pièce moyennement intéressante: ils te réécrivent ce que tu as déjà joué!» (ER, entretien du 13/10/2012).

À l'évidence, le processus de création de Fenêtre Ovale a été traversé de multiples négociations plus ou moins implicites entre le compositeur et les interprètes afin d'atténuer la tension existant entre deux cadres d'interaction potentiellement conflictuels: d'un côté le cadre de la relation compositeur/interprètes, avec la hiérarchie que cela suppose; de l'autre, le cadre de l'improvisation comme processus de création autonome, et donc concurrent du processus compositionnel. Ces négociations débouchent progressivement sur trois principes, destinés à rendre le projet viable en faisant en sorte qu'aucune des parties ne se sente lésée-et notamment que les improvisateurs ne se sentent ni dépossédés ni instrumentalisés:

1. D'abord, la composition d'une partition relativement close, qui n'exige pas des musiciens/interprètes qu'ils improvisent, ceci afin d'éviter toute instrumentalisation des improvisateurs-instrumentalisation qui aurait pu 
être particulièrement mal vécue dans un contexte où, comme nous l'avons vu ci-dessus, c'est le compositeur qui assume, in fine, la responsabilité de l'œuvre et en retire l'éventuel crédit. ${ }^{24}$ Denis Levaillant a bien évoqué les frustrations qui ont pu surgir à l'occasion de certaines œuvres totalement ouvertes (comme Aus den Sieben Tagen de Stockhausen) dans lesquelles le compositeur maintient son autorité tout en confiant aux interprètes la responsabilité effective du déroulé musical: «l'impasse totale de cette "intuition" de collectif, c'est quelle est en effet encore et toujours la projection d'un seul, celui qui signe en fin de compte la partition. [. . .] Des sept 33 tours qui fixent sept improvisations du groupe X, en août 1969, on retient en réalité sept réalisations d'une partition de Karlheinz Stockhausen» (Levaillant 58). C'est typiquement ce genre de malentendu qu'il s'agit ici d'éviter.

2. Ensuite, la composition de miniatures: Fenêtre Ovale repose essentiellement sur des miniatures qui permettent une évidente condensation du geste compositionnel, des transformations rapides de matière, des articulations collectives franches, bref, toutes choses qui sont plutôt difficiles à réaliser dans le contexte de l'improvisation collective libre, qui obéit le plus souvent à des logiques fondamentalement processuelles. C'est d'ailleurs particulièrement vrai dans le cas de ce duo, qui produit une musique assez étirée, qui se développe par mutations et interpolations progressives. Le choix des miniatures permet, en un sens, de légitimer ce que peut être la «valeur ajoutée de la composition» ${ }^{25}$-pour reprendre une expression utilisée par le clarinettiste-par rapport à l'improvisation. ${ }^{26}$

3. Enfin, la composition d'une œuvre utilisant presque exclusivement le matériau sonore du duo, ${ }^{27}$ l'idée étant que les improvisateurs ne se retrouvent pas dans la situation typique de l'interprète devant assimiler des propositions musicales qui lui sont imposées de l'extérieur. ${ }^{28}$

Ce dernier point permet d'ailleurs aux musiciens, quand ils interprètent l'œuvre, de trouver plus facilement cet état d'esprit «improvisatoire» que visent parfois les interprètes (voir Dolan, Sloboda et al. 2013): la proximité avec les gestes et matériaux utilisés est telle que les interprètes peuvent se sentir très libres—d'ailleurs encouragés en cela par le compositeur ${ }^{29}$-ce qui ne peut que renforcer le sentiment d'une réinvention permanente de la musique, à mille lieues d'une reproduction statique, automatisée et figée (voir Clarke, Cook, et al. 2005).

Au delà de ce rapport intime des interprètes au matériau musical utilisé dans Fenêtre Ovale, le compositeur a essentiellement procédé de deux manières pour parvenir à son objectif initial, celui d'une composition qui se rêverait improvisation.

II y a d'abord tout un jeu sur le couple détermination/indétermination, ce qui est à l'évidence une des préoccupations majeures de la réflexion sur la notation depuis les premières partitions graphiques de l'école de New-York. En examinant les esquisses, on peut observer un nombre important d'hésitations et d'allersretours afin de savoir jusqu'à quel point il est nécessaire de déterminer tel ou tel aspect de la notation pour pouvoir satisfaire deux objectifs concurrents:

1. Garantir un certain résultat musical, à la fois fidèle aux intentions du compositeur et reproductible.

2. Autoriser une flexibilité et une souplesse d'interprétation nécessaires pour que la musique possède ce caractère vivant et spontané que peuvent manifester, de manière exemplaire, certaines improvisations.

II s'agit donc, pour le compositeur, de trouver le bon équilibre entre détermination et indétermination. D'une manière générale on remarque que la tendance globale a plutôt été de réintroduire davantage de détermination dans une musique qui pouvait être, initialement, assez indéterminée. Ainsi, dans une des esquisses de «Rondo» (voir figure 13), on voit que le compositeur a un temps envisagé une notation plus graphique, avec des durées relatives, pour obtenir un caractère plus improvisé parce qu'il n'était pas satisfait de l'aspect trop rigide et «solfégique,» selon ses propres mots, provoqué par une notation plus traditionnelle. Finalement, cette qualité a été obtenue en laissant les hauteurs bruitées indéterminées plutôt que les durées. La figure 14 va dans le même sens: on voit dans cette esquisse que le tempo du geste percussif réalisé au piano avec une baguette sur un aimant placé sur une corde était initialement laissé au choix de l'interprète, avant de devenir parfaitement déterminé dans la version finale (croche $=180)$. Ici, le compositeur s'autorise à fermer cet espace d'indétermination parce qu'il s'est rendu compte que le type de geste réalisé à la clarinette (qui joue avec le pavillon à fleur d'eau pour produire une très légère ondulation) suffit à imposer un temps «naturel,» étroitement dépendant des conditions de la performance. Cette tendance générale (d'une musique initialement peu déterminée à une partition très précise, aux paramètres compositionnels relativement déterminés) se comprend d'autant mieux si l'on se rappelle que le projet compositionnel global s'est progressivement redessiné au fur et à mesure du travail collectif, d'un objectif «comprovisatoire» à la réalisation d'une partition obéissant largement à la dichotomie composition/interprétation, même si l'improvisation-et c'est là tout notre propos—a joué un rôle particulièrement explicite dans cette réalisation. 


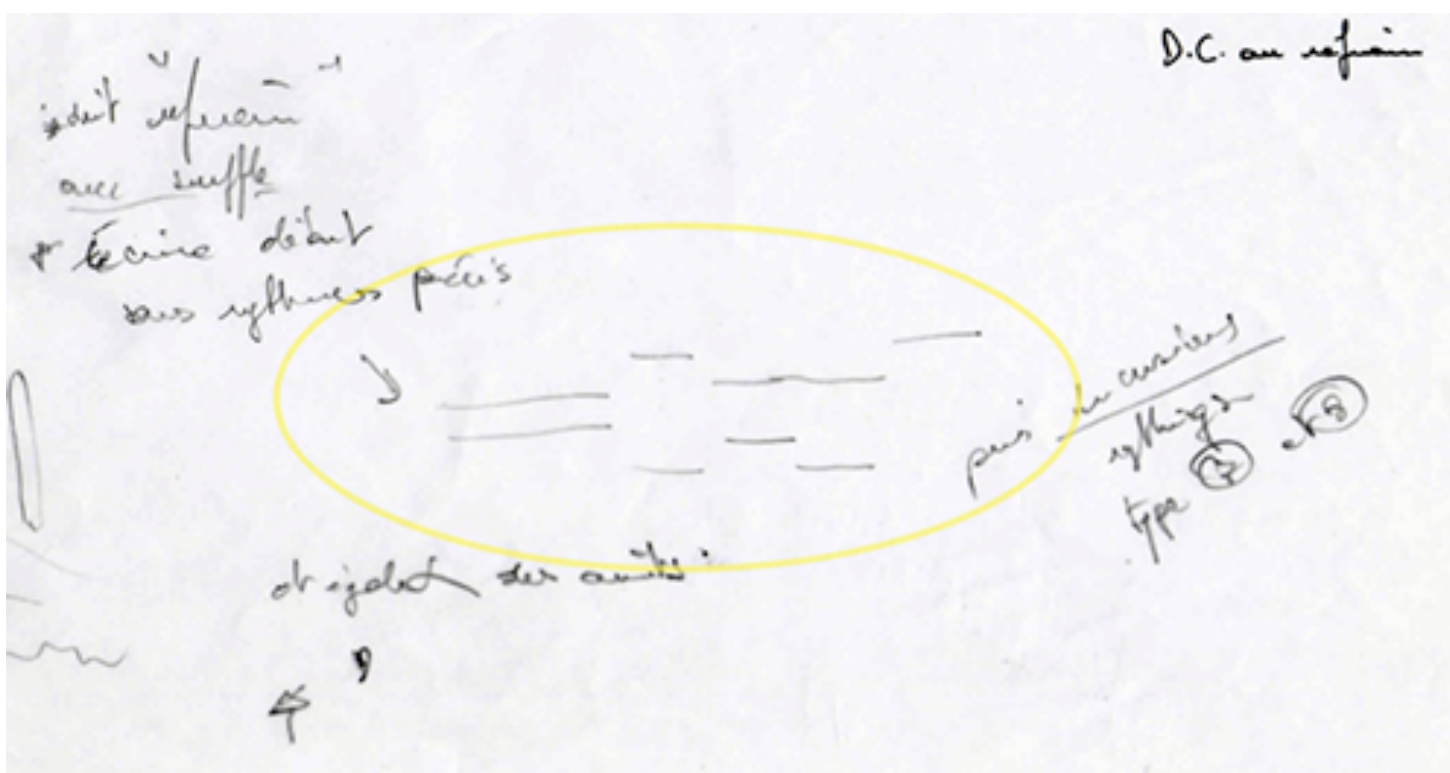

Rowso $3 / 3$

Figure 13. Une esquisse de «Rondo,» dans laquelle le compositeur envisage une notation rythmique proportionnelle.

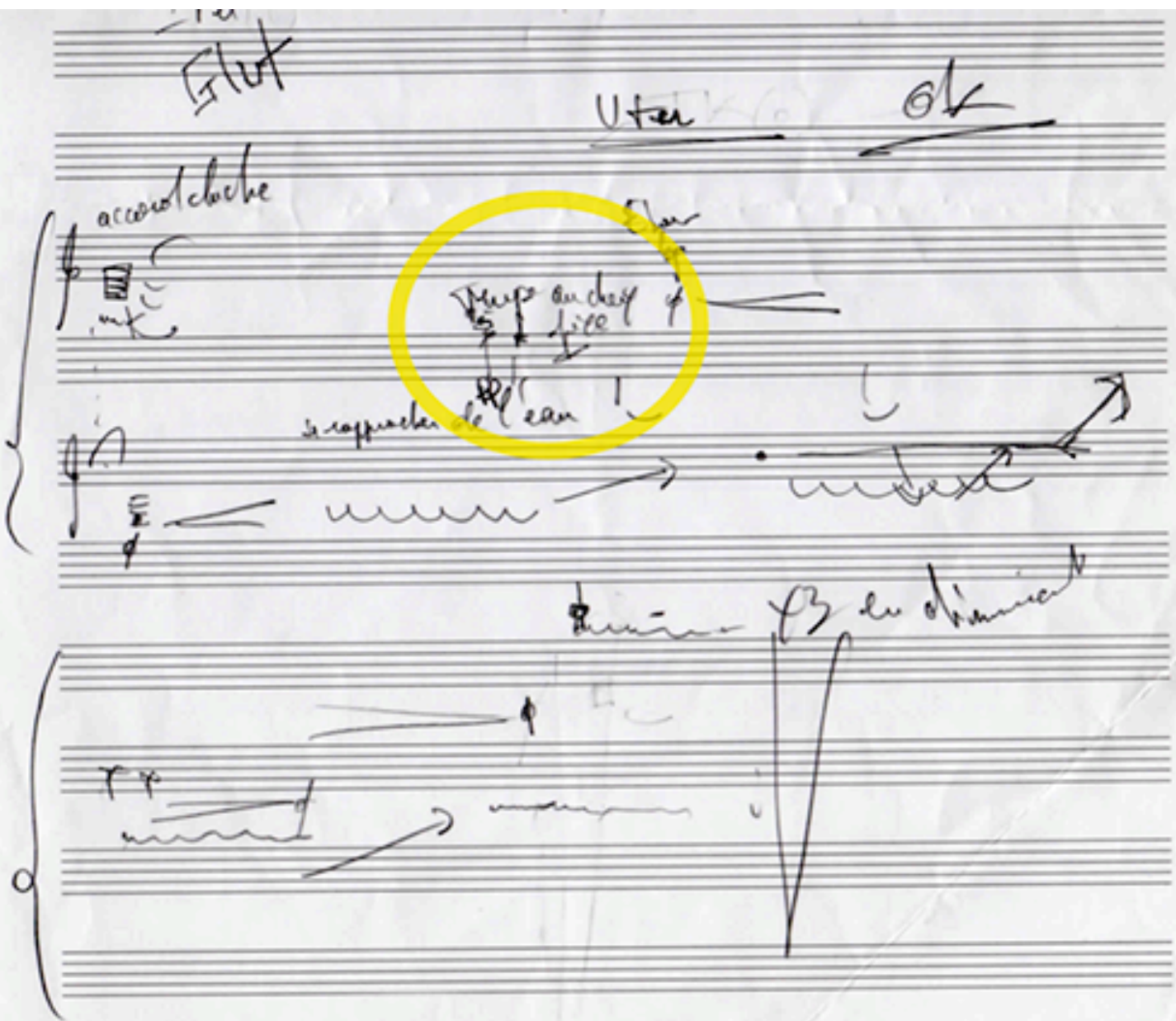

Figure 14. Première esquisse de «In Aquam,» laissant la pianiste libre de son tempo. 
De manière générale, ce n'est donc pas en adoptant une écriture très indéterminée que le compositeur entend capturer un sentiment improvisatoire, mais plutôt en choisissant de laisser systématiquement un paramètre de libre: «en fait je me suis rendu compte que c'est souvent dans l'effacement d'une détermination qui ne servait à rien que se passait la possibilité de trouver une souplesse» (KN, entretien du 26/09/2012). Et en parcourant les différentes pièces, on retrouve effectivement ce principe, décliné sur différents paramètres en fonction du contexte musical: indétermination des durées et donc placement relatif des musiciens l'un par rapport à l'autre dans la pièce «Khen»; choix des hauteurs dans un réservoir dans la pièce éponyme «Fenêtre Ovale»; répétitions ad libitum dans «Kroum»; gestes réalisés à l'intérieur du piano relativement peu précisés en termes de hauteur dans «Tremuli»; etc.

Mais il y a plus important encore, et sans doute bien plus spécifique à la démarche de Fenêtre Ovale: pour capturer ce sentiment d'improvisation, le compositeur mobilise également un nombre important de ces gestes fragiles, instables, transitoires, en équilibre vacillant entre deux états, avec lesquels ce duo travaille tout particulièrement, qui non seulement réintroduisent une certaine dimension d'imprévisibilité dans la musique, mais qui encore nécessitent un temps intrinsèque pour effectivement se préciser, se stabiliser, se définir; cela renforce évidemment le sentiment de présence que l'on peut avoir à l'écoute de cette musique, c'est-à-dire le sentiment d'une musique créée sous nos oreilles. Ces gestes fragiles, en créant leur propre temporalité, forcent et obligent les musiciens à réagir en fonction de ce qui va se passer dans le moment du jeu. C'est d'ailleurs quelque chose d'assez général dans cette œuvre: la partition étant fondée sur des phénomènes d'interaction sonore, ce sont souvent des rapports de gestes qui fixent la temporalité. La pièce «In Aquam» (voir figure 15) en fournit un exemple parfait: la fragilité intrinsèque du geste inaugural du clarinettiste-qui tente de faire apparaître des remous audibles en approchant très progressivement le pavillon d'une bassine d'eau-dicte en réalité sa temporalité aux interventions de la pianiste et, partant, impose un déploiement temporel des matières sonores «naturel», étroitement dépendant des conditions de performance de la pièce tout entière (voir extrait son ore 6). On pourrait faire une remarque semblable à propos de la fin de «Tremuli» - qui fait alterner des états de matière très fragiles (la vibration d'une feuille de calque sur les cordes graves du piano, un sifflement suraigu de clarinette, des crissements produits par un carreau de verre glissé sur les cordes, un son de souffle à la clarinette qui devient très progressivement flatt de gorge, etc.) —et, à vrai dire, d'un très grand nombre de pages de Fenêtre Ovale.

Cette importation de gestes dérivés du travail du duo Risser/Rühl dans un contexte composé est en réalité au cœur du travail de «capture» de l'improvisation qui s'opère dans le processus de création de Fenêtre Ovale. C'est en effet en construisant la musique autour de ces gestes-et en élaborant avec les musiciens une notation suffisamment souple et ouverte pour leur permettre de remobiliser naturellement leurs gestes d'improvisateurs dans une situation d'interprétation ${ }^{30}$ - que le compositeur entend retrouver quelque chose de l'improvisation dans son œuvre, et satisfaire l'idéal régulateur, au cœur du projet Fenêtre Ovale, d'une composition qui «sonne» comme une improvisation. 


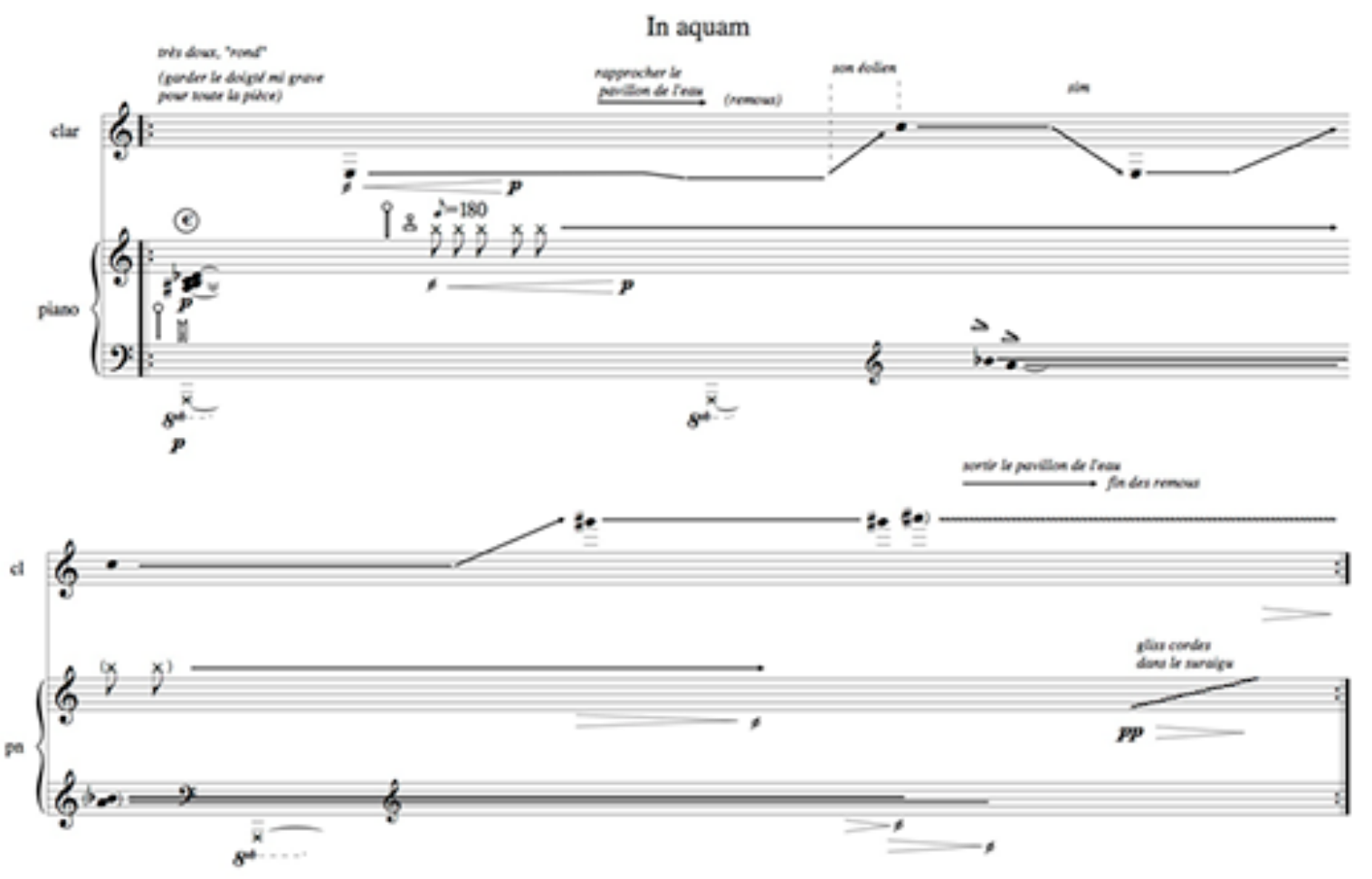

Figure 15. «In Aquam»

Toutefois, cette absorption des gestes du duo Risser/Rühl par la partition de Karl Naëgelen ne va pas de soi. Et les tensions inhérentes à une telle démarche permettent assurément de révéler, a contrario, ce qui fait la spécificité respective des gestes de l'improvisateur et du compositeur.

D'abord, s'il fallait caractériser la forme d'inventivité qui est à l'œuvre dans l'exploration instrumentale de ce duo d'improvisateurs, on pourrait dire qu'elle ressortit d'une forme de bricolage, dans laquelle les moyens instrumentaux se trouvent articulés à une pragmatique musicale: nous avons affaire à une recherche empirique, pas forcément rationalisée, mobilisant volontiers des objets trouvés (la diversité et, parfois, l'incongruité des objets utilisés par la pianiste sont à cet égard parfaitement révélateurs), qui vise à observer et à inventorier les liaisons ou rapports entre geste et son qui soient mobilisables dans une situation de jeu, avec une certaine contrainte d'efficacité et d'adaptabilité, liée à l'impératif de production en temps réel.

Cette connaissance «de l'intérieur» des différents gestes instrumentaux contraste avec l'esprit de système «extérieur» du compositeur qui cherche plutôt à épuiser les différentes facettes d'un même geste, en systématisant ces diverses explorations pour en isoler les différents paramètres, en analyser le mode de fonctionnement et, au final, en garantir la reproductibilité. ${ }^{31}$

Ce travail de systématisation s'est principalement opéré dans l'étape de catalogage des techniques instrumentales des musiciens et de constitution de la sonothèque mentionnée ci-dessus. L'examen de cette sonothèque permet de se rendre compte de deux éléments très importants:

1. Même dans cet exercice de catalogage-dont on pourrait imaginer qu'il vise à identifier le plus précisément possible un mode de jeu, la relation entre un geste donné et un son donné-le rapport du musicien improvisateur à son instrument est déjà clairement exploratoire: sur une technique donnée, il essaye de montrer comment celle-ci peut évoluer, la manière dont elle est transformable, et toujours ductile. Le geste est pensé dans une dimension dynamique, en mouvement, porteur d'une évolution et d'un cheminement musical potentiels-conformément à son utilisation en situation d'improvisation-et jamais comme un objet réifié, aux composantes bien isolées. On pourra s'en faire une idée en se reportant à l'extrait sonore 7, dans lequel le clarinettiste expose un mode de jeu au compositeur, exposition qui se transforme vite en une présentation d'un travail de métamorphose graduelle du timbre, en utilisant plus ou moins de salive, à partir d'un même doigté de mi grave. 
2. C'est ensuite dans un deuxième temps que le compositeur cherche à systématiser cette exploration. L'extrait sonore 8 fait ainsi entendre le travail que le compositeur a demandé au clarinettiste, à partir d'un multiphonique souvent utilisé par celui-ci en situation d'improvisation, ${ }^{32}$ et qui servira de base à la pièce «Tremuli»:

Donc c'est un multiphonique très piano, avec un grand nombre d'harmoniques (y compris très aigues); je n'y aurais sans doute pas pensé moi-même, mais je me rappelle que, dans le travail, Karl me disait: «Est-ce que tu arrives à isoler seulement les deux hauteurs les plus aigues et à faire un trille avec? Est-ce que tu arrives à faire ressortir la note grave mais sans sortir une note intermédiaire?» Et moi je n'ai jamais pensé à faire un travail aussi systématique: donc il me propose, j'essaye, je dis «tiens, ça marche» et on continue... (JR, entretien du 17/09/2012)

Cette approche très systématique des différentes possibilités de trille à partir d'un multiphonique donnéobjectivation seule à même de garantir la reproductibilité du geste-est à mille lieues du rapport éminemment temporalisé qu'entretiennent bon nombre d'improvisateurs avec leurs gestes instrumentaux, toujours pensés comme faisant partie prenante d'un processus dynamique.

Or le travail du compositeur dans les miniatures de Fenêtre Ovale a précisément consisté à découpler les gestes des improvisateurs de leur contexte dynamique, soit en les condensant extrêmement (par exemple en enchaînant deux gestes musicaux qui, dans le contexte de l'improvisation, sont prétextes à une lente interpolation), soit en les réarticulant (en proposant des manières inédites, et donc en un sens artificielles, de combiner ces gestes). On touche là à un des paradoxes évidents de la démarche qui est au cœur du travail de Fenêtre Ovale: la capture des gestes de l'improvisateur par le compositeur suppose ce processus d'objectivation et de systématisation que nous venons de décrire; mais, par là même, il perd ce qui fait une des spécificités de ce geste, à savoir sa dimension toujours dynamique et contextuelle. Ce découplage produit évidemment un effet de mise à distance des geste instrumentaux de l'improvisateur, pourtant intrinsèquement liés à son identité même, et ce sous de multiples aspects: identité corporelle, identité artistique, identité esthétique, identité professionnelle... bref, tout ce qui fait la singularité du musicien improvisateur. Cela nous invite à mettre en lumière deux tensions supplémentaires inhérentes à ce genre d'approche.

La première tension, c'est celle qu'il y a entre l'abstraction que suppose le travail de généralisation par la notation et la dimension profondément idiosyncrasique des gestes de l'improvisateur. D'un côté, celui de l'improvisateur, on a une compréhension physique, gestuelle du son: un son, c'est une manière de faire sur l'instrument; de l'autre côté, celui du compositeur, comprendre ce son, c'est le rationaliser, l'abstraire dans une certaine mesure de son incarnation spécifique, pour le généraliser par la notation, ce qui suppose de découpler le geste du son. Dans la plupart des cas, ce que le compositeur cherche à noter, à capturer, c'est une image acoustique: le geste n'est là que pour dire comment parvenir à cette image acoustique. Même si les tentatives pour penser différemment le rapport geste/notation sont évidemment innombrables, l'écriture semble être condamnée à rester toujours sous-déterminée, et à ne pouvoir prendre en compte toutes les singularités corporelles des interprètes, qui devront de toutes façons trouver une manière propre d'ancrer corporellement ce geste, en fonction du résultat sonore. Ici, le passage par le filtre de la notation a transformé ce rapport intime entre son et geste même pour les improvisateurs à l'origine de l'œuvre, qui ont parfois fini par devoir trouver eux-mêmes de nouveaux gestes pour réaliser ce qui est noté (alors que ce qui est noté venait précisément de leurs propres gestes d'improvisateurs). En effet, dès que les musiciens se placent dans la position d'interprète de cette notation, ce n'est plus le geste instrumental qui est moteur du discours musical mais bien une certaine image acoustique précise qu'il faut viser et atteindre à coup sûr. Le passage par la notation conduit ainsi rétroactivement les improvisateurs à modifier leurs propres gestes instrumentaux, alors qu'ils sont, d'une certaine façon, les auteurs des techniques proposées initialement, et capturées par le compositeur. ${ }^{33}$

La seconde tension, c'est celle qu'il y a entre l'explicitation que suppose le travail de standardisation par la notation et la dimension profondément singularisante des gestes de l'improvisateur. Le passage par la partition est une explicitation: entre la notice détaillant les divers modes de jeu utilisés, la nomenclature listant les différents objets utilisés pour la préparation du piano, et les pièces elles-mêmes, la partition de Karl Naëgelen se rapproche à certains égards du « mode d'emploi »: elle exhibe des techniques, révèle comment obtenir tel ou tel son, comment parvenir à tel effet, à telle texture... ${ }^{34}$ Or cet artisanat est précisément ce qui contribue, en partie, à forger l'identité de l'improvisateur: l'écriture de la partition peut alors agir comme une dépossession, au sens propre, de l'improvisateur. Certaines remarques de la pianiste sont à cet égard tout à fait révélatrices de cet aspect problématique de la collaboration:

II y a des choses que je ne voulais pas dévoiler, parce que c'était des sons trop personnels: c'est comme si je devais dire... C'était très intime [. . .]. Avec Joris on travaille sur la magie sonore: si tu dis ton truc de magie, ça ne marche plus! Je ne vais pas dévoiler mes tours de magie pour que d'autres deviennent pseudo-magiciens alors qu'ils n'ont pas travaillé la question. À la limite, c'est pas grave qu'ils imitent: mais moi, ça m'enlève une part de ma magie [. . .]. Mais je crois que j'ai une 
petite déontologie: je ne veux pas piquer les trucs des autres alors je ne veux pas qu'on vienne me piquer les miens. [. . . ] Et puis c'est quand même mon gagne-pain. L'improvisation c'est mon premier métier: c'est là-dessus que je vis». (ER, entretien du 13/10/2012)

Finalement, on est renvoyé ici à cette crainte déjà ancienne de la fixation ou de l'objectivation de l'improvisation (sous la forme d'une partition ou d'un enregistrement ${ }^{35}$ ) qui, en dépossédant l'improvisation de son aura, dépossèderait également l'improvisateur de sa singularité musicale, de ce qui le caractérise en propre et le démarque des autres. Que cette angoisse soit toujours présente est peut-être le signe d'une antinomie plus profonde, entre l'essence processuelle de l'improvisation, et le mouvement de réification que présuppose une certaine pratique de la composition, et d'une tension sans doute irréconciliable entre la visée généralisante du geste compositionnel ${ }^{36}$ et la dimension au contraire profondément singularisante du geste de l'improvisateur.

\section{Conclusion}

À travers son paradoxe constitutif-une collaboration entre un compositeur et des improvisateurs qui débouche sur une œuvre dans laquelle il n'y a pas la moindre improvisation-Fenêtre Ovale nous a permis de poser une série de questions dont la portée dépasse largement le cas particulier de l'œuvre considérée:

1. Quelle place pour l'improvisation dans le processus de composition?

2. Par quelles modalités d'écriture simuler l'improvisation?

3. Dans quelle mesure les gestes de l'improvisateur peuvent-ils se laisser absorber par la composition?

La reconstitution du processus de création de Fenêtre Ovale apporte quelques réponses-certes encore embryonnaires-à ces questions. En particulier, notre analyse a fait apparaître deux éléments importants:

1. L'improvisation a pu occuper trois fonctions essentielles tout au long du processus compositionnel: une fonction de stimulation, les trouvailles timbrales et instrumentales propres au travail d'improvisateur irriguant une esthétique de l'illusion sonore qui est au cœur de Fenêtre Ovale; une fonction d'inspiration, les matières et idées musicales apparues dans le temps de l'improvisation se trouvant remobilisées, de diverses manières, dans l'écriture de l'œuvre; et une fonction de simulation, l'improvisation venant compléter un état encore lacunaire de la partition ou fournir de toute pièce une représentation sonore d'un processus formel encore indéterminé, ce qui permet au compositeur de valider ou d'invalider un certain nombre de choix.

2. Le processus de création de Fenêtre Ovale peut être lu comme un processus d'absorption de l'improvisation par la composition-l'improvisation comme horizon rêvé de la composition—qui se fait essentiellement par la capture d'un certain nombre de gestes instrumentaux et musicaux issus du travail des improvisateurs, à même de véhiculer quelque chose de la présence de l'improvisation.

Mais notre étude a également permis de révéler les limites et paradoxes intrinsèques d'une telle démarche. II semble en effet y avoir une série de tensions difficilement réconciliables entre le geste du compositeur et le geste de l'improvisateur, tels qu'ils nous sont donnés à voir dans le cadre de la création de cette œuvre: tension entre le geste du compositeur visant à l'objectivation systématisée et décontextualisée du rapport à l'instrument et le geste de l'improvisateur, toujours profondément temporalisé et inséparable d'une certaine logique dynamique (transformation, évolution, mutation...); tension entre le geste du compositeur visant à généraliser un rapport à l'instrument par son inscription au sein d'une notation partageable et le geste de l'improvisateur, toujours profondément idiosyncrasique; tension, enfin, entre le geste du compositeur visant à expliciter un rapport à l'instrument, alors que ce rapport à l'instrument revêt une dimension profondément singularisante pour l'improvisateur, source de son identité à la fois artistique et professionnelle.

Ce n'est pas le moindre des mérites des musiciens impliqués dans la création de Fenêtre Ovale que d'avoir su mettre en place, progressivement, les modalités d'une collaboration qui, bien que s'inscrivant assez clairement dans le cadre d'une relation compositeur/interprètes, ne débouche pas sur une dépossession pure et simple des improvisateurs mais, au contraire, parvienne à faire surgir une réponse originale et excitante à la rencontre-toujours un peu incertaine-de l'improvisation et de la composition. 


\section{Notes}

${ }^{1}$ C'est la thèse célèbre de Lydia Goehr sur le rôle régulateur du concept d'œuvre: «Vers 1800, quand la composition fut définie comme impliquant la prédétermination du plus grand nombre possible d'éléments structurels, la notion d'improvisation acquit sa signification moderne. Pour la première fois, on la considérait comme s'opposant strictement à la composition au sens propre» (Goehr 234).

${ }^{2}$ On peut penser tout particulièrement au foisonnement de dispositifs informatiques interactifs, véritables «partitions fantômes» (pour reprendre un terme de Morton Subotnick) venant cadrer les improvisations des solistes (voir Dudas 2010 ou le projet Native Alien du compositeur Sandeep Bhagwati).

${ }^{3}$ Voir l'essai de Jerrold Levinson, «Hybrid Art Forms» (1984).

${ }^{4}$ Même si ce n'est pas forcément le cas, comme le montre bien le travail de Donin et Féron (2012) sur la composition de Gramigna de Stefano Gervasoni.

${ }^{5}$ Dans une étude empirique récente, Ulla Pohjannoro (2014) a montré comment le processus créatif du compositeur oscillait constamment entre une génération intuitive d'idées et une génération réflexive.

${ }^{6}$ Voir par exemple l'étude ethnographique réalisée sur l'œuvre de Liza Lim, Tongue of the Invisible, avec le pianiste de jazz Uri Caine et l'ensemble musikFabrik (Clarke, Doffman et Lim, 2013) qui, pour mettre en avant la distribution de la créativité à l'œuvre dans ce processus musical, se penche précisément sur les étapes finales du travail—quand il s'agit déjà de réaliser l'œuvre—à quelques semaines de la création.

${ }^{7}$ Citons la violoncelliste Frances-Maris Uitti avec Jonathan Harvey, le pianiste Volker Banfield avec György Ligeti, le clarinettiste Armand Angster avec Pascal Dusapin...

${ }^{8}$ Notre méthodologie n'est évidemment pas la plus adaptée pour étudier précisément la distribution de la créativité qui s'opère dans le processus de création de Fenêtre Ovale. L'analyse fine du réseau d'interactions existant entre le compositeur et les improvisateurs aurait supposé une approche ethnographique (à l'instar de Clarke, Doffman et Lim 2013)—à même de saisir in vivo la cristallisation et l'évolution de ces interactions-plutôt que l'approche de reconstitution a posteriori qui est la nôtre ici. Toutefois, le croisement des entretiens semidirectifs menés avec les trois protagonistes nous a tout de même permis de retracer dans ses grandes lignes l'évolution du rapport entre le compositeur et les interprètes. ${ }^{9}$ Voici un extrait de la note d'intention rédigée alors que Fenêtre Ovale n'était encore qu'à l'état de projet: «Nous
avons l'ambition d'innover par rapport à un tandem 'compositeur-interprète' traditionnel. En effet, l'idée que nous
nous faisons du travail que nous mènerons ensemble s'approche de celui que pratiquent certains danseurs ou
performers, pour lesquels la création n'est pas possible sans une période importante de recherche, intense et
collective. Dans un monde musical souvent gouverné par un rapport productiviste au travail, nous souhaitons
introduire, grâce au temps important que nous pourrons investir, de l'improvisation, des tâtonnements, des
essais, des retours en arrière. C'est seulement à ce prix que la formulation 'confrontation de démarches' peut
avoir un sens: en constituant petit à petit une expérience commune, en systématisant les allers-retours entre
propositions et réaction.»

${ }^{10}$ «Moi j'ai eu ces envies [de parfois modifier le texte], mais en réalité on se retrouve plutôt dans la situation d'interprète où on se dit: 'si ça résiste, c'est qu'il faut trouver la voie pour en faire quelque chose de bien'» (Joris Rühl, entretien du 17/09/2012).

${ }^{11}$ «C'est lui qui a dirigé ces séances; il avait des questions précises par rapport aux improvisations qu'on avait faites. C'est lui qui disait: 'Tiens, je me rappelle que vous aviez fait ça, est-ce que tu peux me refaire tel son?'» (Joris Rühl, entretien du 17/09/2012).

12 «Des espaces ouverts qui se sont fermés; d'autres qui se sont ouverts, mais globalement c'est plutôt le contraire» indique le compositeur à propos de l'évolution au fil du temps des différentes pièces de Fenêtre Ovale (Karl Naëgelen, entretien du 24/02/2013).

${ }^{13}$ «Du coup je regrette parce que... le projet s'est quand même étalé sur plus d'un an, donc il y a eu des allersretours, il a réajusté des choses après nous avoir entendu jouer, mais je trouve qu'il y aurait pu avoir plus d'allers-retours; moi j'aurais souhaité qu'à partir de ses propositions, on ait encore une exploration qui puisse amener à... Je ne suis pas du tout fâché par contre que ce soit très déterminé—une musique très écrite-mais je me dis qu'il y aurait peut-être pu avoir un peu plus d'allers-retours» (JR, entretien du 17/09/12). 
14 «Quand l'idiome vient à manquer, on peut aller au matériau-où pouvoir est à entendre à la fois comme possibilité et comme autorisation: on peut, c'est-à-dire 'on a la possibilité de' et 'alors seulement on peut'» constate ainsi l'improvisateur Jean-Luc Guionnet (Guionnet 131).

${ }^{15}$ C'est ce que Bernard Sève (2013) appelle l'archi-écriture de l'instrument, l'ensemble des choix organologiques et des déterminations acoustiques qui sont déjà la trace d'une certaine compréhension du musical.

${ }^{16}$ Split LP_Risser/Rühl_OBLIQ \& Christof Kurzmann, Umlaut Records, 2010, UB003.

17 «L'idée de départ de 'Tremuli'-c'est un peu l'idée de départ de tout le cycle, en fait—c'était de créer des objets harmoniques où la confusion des sources est vraiment totale» (KN, entretien du 24/02/2013).

${ }^{18}$ On citera pêle-mêle: le travail d'archet du contrebassiste Stefano Scodanibbio; le travail sur les multiphoniques du duo norvégien Streifenjunko (trompette/saxophone); la voix d'Isabelle Duthoit; ou encore les guitares préparées de Sharif Sehnaoui. . .

19 «Le timbre et l'improvisation, c'est complètement lié pour moi. J'ai remarqué que les improvisateurs-en tout cas ceux que j'ai rencontrés-avaient développé un rapport au sonore qui était poussé, qui était travaillé. Dans le cas de Joris ou de Ėve, c'est vrai que la recherche sur des sons sur leurs instruments, c'est leur quotidien. [. . .] C'est du pain béni pour un compositeur d'avoir une palette de sons à disposition, comme un peintre pourrait avoir une palette de couleurs» (KN, entretien du 26/09/2012).

${ }^{20}$ Cet aspect du rapport entre improvisation et composition est particulièrement évident chez un compositeur comme Giacinto Scelsi. Beaucoup de ses œuvres sont nées d'improvisations réalisées par Scelsi lui-même, au piano ou sur ses ondiolas, dont les bandes étaient ensuite confiées, parfois accompagnées de plans de montage sommaires à un copiste qui avait alors la charge de coucher sur papier à musique le contenu sonore.

${ }^{21}$ «En fait, je n'étais pas très sûr de mon coup... Je suis venu avec une idée de refrain, mais que je voulais bruitiste. Donc je l'ai écrit rythmiquement, comme ça, à peu près. Mais les modes de jeu n'étaient pas du tout précis. Et puis mon but c'était de venir avec quelque chose pour pouvoir tester des choses, et puis dans un deuxième temps de repréciser. [. . . J Je pense qu'on a essayé différents souffles: Ėve avec ses velours, Joris aussi, différents types de souffles à la clarinette, et puis j'ai dû en choisir certains à ce moment-là. Tiens, par exemple 'souffle-tissu avec attaques' [voir Figure $7,3^{\mathrm{eme}}$ système, partie de piano], ce n'est pas la même écriture que le reste: je pense que c'est quelque chose que j'ai rajouté suite à leurs propositions» (KN, entretien du 24/02/2013).

22 «À partir de là, je ne savais pas trop quoi faire, en gros. Donc ça se développe, et puis effectivement I'harmonie est complétée en traits. En fait c'est typiquement un endroit où je n'étais pas bien sûr de ce que ça allait devenir» (KN, entretien du 24/02/2013).

${ }^{23}$ Le compositeur, qui ne se souvenait plus du tout de cette étape du travail, nous a confirmé cette hypothèse: «En fait j'étais dans l'idée de me demander si ce truc-là pouvait revenir... si c'était un matériau qui pouvait s'opposer au matériau de ce qui allait devenir 'Tremuli' et permettre de revenir au matériau de 'Tremuli,' et donc de faire partie de la même pièce. Je pense que c'était une contrainte [d'improvisation] pour voir si c'était un matériau qui résistait trop ou si c'était un matériau qui pouvait aller dans cette direction. Finalement, la décision a été de faire un morceau à part. C'est typiquement un moment où, comme je ne suis pas sûr de mon coup, je leur demande d'improviser! Je me demandais si ça pouvait basculer vers autre chose et j'ai demandé un truc de forme, et ils ont essayé de le faire» (KN, entretien du 21/05/2013).

24 «Quand on dit au musicien: 'tu passes de là à là par l'impro[visation],' on lui dit: 'improvise, mais quand même, tu me fais une belle transition!' En fait, ça met l'improvisateur dans une position de semi-improvisation que la plupart n'aiment pas beaucoup» (KN, entretien du 26/09/2012).

${ }^{25}$ «Karl nous écoute et nous dit: 'c'est vraiment bien,' et il avait l'inquiétude de ne pas... enfin il se disait: 'quelle est la valeur ajoutée de la composition?', ou quelque chose comme ça. Nous, on fait plutôt des choses longues, formellement; et c'est pour ça qu'il a écrit des miniatures. Et, du coup, non seulement à l'échelle des pièces, c'est beaucoup plus ramassé, mais aussi à l'échelle des gestes, des ruptures... Là, je pense que c'est assez différent, c'est vraiment sa patte. II y a des choses qui ne durent qu'une ou deux secondes et qu'on ne ferait pas du tout en situation d'improvisation» (JR, entretien du 17/09/2012). 
${ }^{26}$ «À un moment, il a fallu que je reprenne confiance pour me dire que, moi, j'allais aussi leur apporter quelque chose. Quand on a écouté Joris et Ėve en tant que duo d'improvisation, souvent c'est des moments qui prennent du temps, avec des processus longs à changer, etc. Moi j'arrive, je suis compositeur, qu'est-ce que j'ai envie de faire? Forcément, je taille dans l'histoire et puis je propose quelque chose qui est très spécifique de... qu'on peut certainement obtenir en tant qu'improvisateur mais, enfin, qu'il est plus facile d'obtenir en tant que compositeur» (KN, entretien du 26/09/2012).

${ }^{27}$ II y a deux exceptions: l'utilisation du khen dans une des pièces, qui ne fait pas partie de l'instrumentarium habituel du duo; et le matériau musical de «Kroum»-d'ailleurs une des premières idées notées par le compositeur-qui évoque beaucoup plus directement l'univers habituel de Karl Naëgelen («Kroum, ce n'est pas notre musique»; ER, entretien du 13/10/2012).

${ }^{28}$ «L'approche pour monter les pièces, par rapport à ce que j'ai pu faire par ailleurs en musique contemporaine, était quand même assez différente. Je connais bien les sons, ce sont mes sons: on a quasiment vu ensemble comment il allait les écrire, et je ne me suis pas retrouvé comme ça m'est déjà arrivé avec une phase de plusieurs heures de travail très laborieux, pour jouer un truc et ne comprendre qu'a posteriori comment ça sonne ou comment le compositeur veut que ça sonne» (JR, entretien du 17/09/2012).

${ }^{29}$ «Karl nous encourage beaucoup à être spontanés; je ne vais pas dire à désobéir... mais il n'est pas du tout avec nous dans une démarche autoritaire» (JR, entretien du 17/09/2012).

${ }^{30}$ Cette indétermination dans l'écriture permet de respecter l'organicité du geste de l'improvisateur, habitué à réaliser spontanément certains enchaînements corporels dans le temps de l'improvisation. La pianiste Ėve Risser est revenue explicitement sur ce point: «ça m'arrangeait qu'il [le compositeur] écrive des sons que je fais déjà 'organiquement': par exemple, si je suis en train de faire quelque chose sur le clavier et que je dois aller caoutchouter le milieu du piano, s'il m'écrit quelle note, ça va être difficile. Mais s'il me met un petit trait pour dire 'fais ça, à ce moment-là,' moi je vais avoir l'habitude, parce que je le fais déjà souvent en jouant. II y a parfois des gestes qu'il a été pratique de ne pas trop préciser» (ER, entretien du 13/10/2012).

${ }^{31}$ Les improvisateurs peuvent certes chercher également cette reproductibilité, pour par exemple garantir la pérennité d'un effet d'une performance à l'autre, mais il faut bien voir que celle-ci entre souvent en conflit avec l'exigence de spontanéité de l'improvisation. Comme le rappelle Joris Rühl: «C'est à la fois un sentiment que je déteste: vouloir un son et que ça ne marche pas; donc je sais bien pourquoi je cherche à les contrôler. Mais j'ai pas tranché: je suis attiré aussi par la 'vraie' improvisation (quand je travaille avec des gens comme Michel Doneda, dont la musique est tellement vivante), où tout peut se passer à tout moment, et ne pas être prisonnier de sa technique» (JR, entretien du 17/09/2012).

32 «Je suis parti d'un multiphonique qu'il avait dans les doigts et qu'il utilisait souvent» (KN, entretien du 26/09/2012).

${ }^{33}$ «Sur "Tremuli", par exemple, je m'écrivais des choses pour garantir au mieux le son: descendre un peu le menton, pincer moins, mettre la langue un peu en arrière... alors que c'est un multiphonique que je fais souvent en improvisation!» (JR, entretien du 17/09/2012).

${ }^{34}$ C'est évidemment particulièrement gênant dans le cas du duo Risser/Rühl, qui développe une esthétique du trompe l'œil et de l'illusion. Cette musique, qui donne à entendre ce que l'on ne voit pas, et qui donc refuse toute «indicialité,» joue sur les mêmes mécanismes qu'un spectacle de prestidigitation. Or la connaissance du «tour de magie,» en donnant accès aux médiations (à la fois ressources cachées et séries de gestes extrêmement rapides), ruine le trouble du spectateur, et le plaisir qui peut en résulter. D'une certaine façon, c'est la "recette» du tour de magie que constituent les improvisations du duo Risser/Rühl qui se trouve dévoilée par la notice de la partition.

${ }^{35}$ On en trouvera une évocation poétique dans le film de Woody Allen, Sweet and Lowdown, dans lequel le personnage principal, le guitariste de jazz imaginaire Emmet Ray, refuse une séance d'enregistrement de peur qu'on lui vole ses "plans.» Voir également cette remarque de l'ethnologue Paul Berliner: "certains musiciens gardent pour eux leurs meilleurs idées durant les sessions d'enregistrement, pour les rendre moins accessibles aux imitateurs» (Berliner 105).

${ }^{36}$ Même lorsqu'un compositeur écrit spécifiquement pour un interprète donné-comme la Sequenza III de Luciano Berio pour Cathy Berberian, qui conduit sans doute «à la limite du reproductible» (Deliège 483)—il y a l'idée que les performances de l'interprète en question, pour idiosyncrasiques qu'elles soient, peuvent servir de modèle. 


\section{Ouvrages Cités}

Bailey, Derek. L'improvisation: sa nature et sa pratique dans la musique. Paris: Outre Mesure, 1999. Imprimé.

Bayley, Amanda et Michael Clarke. Evolution and collaboration: the composition, rehearsal and performance of Finnissy's Second String Quartet. Palatine, 2011. DVD.

Born, Georgina. «On Musical Mediation: Ontology, Technology and Creativity.» Twentieth-Century Music 2 (2005): 7-36. Imprimé.

Benson, Bruce. The Improvisation of Musical Dialogue: A Phenomenology of Music. Cambridge: Cambridge UP, 2003. Imprimé.

Berliner, Paul F. Thinking in Jazz: The Infinite Art of Improvisation. Chicago: The U of Chicago P, 1994. Imprimé

Bertinetto, Alessandro. «Improvisation and Artistic Creativity.» Proceedings of the European Society for Aesthetics 3 (2011): 81-103. Imprimé.

Brown, Lee B. «'Feeling My Way': Jazz Improvisation and Its Vicissitudes. A Plea for Imperfection.» The Journal of Aesthetics and Art Criticism 58.2 (2000): 113-123. Imprimé.

Clarke, Eric. «Creativity in Performance.» Musical Imaginations: Multidisciplinary Perspectives on Creativity, Performance and Perception. Ed. Daniel Hargreaves, Dorothy Miell et Raymond MacDonald. Oxford: Oxford UP, 2011. 17-30. Imprimé.

Clarke, Eric, Nicholas Cook, Bryn Harrison, et Philip Thomas. «Interpretation and Performance in Bryn Harrison's Être-temps.» Musicae Scientiae 9 (2005): 31-74. Imprimé.

Clarke, Eric, Mark Doffman, et Liza Lim. «Distributed Creativity and Ecological Dynamics: A Case Study of Liza Lim's Tongue of The Invisible.» Music and Letters 94.4 (2013): 628-663. Imprimé.

Dahlhaus, Carl. «Qu'est-ce que l'improvisation musicale?» Tracés 18 (2010): 181-196. Imprimé.

Deliège, Célestin. Cinquante ans de modernité musicale: de Darmstadt à l'Ircam. Sprimont: Mardaga, 2003. Imprimé.

Dietrich, Arne et Riam Kanso. «A Review of EEG, ERP and Neuroimaging Studies of Creativity and Insight.» Psychological Bulletin 136.5 (2010): 822-848. Imprimé.

Dolan, David, John Sloboda, Henrik Jensen, Björn Crüts, et Eugene Feygelson. «The Improvisatory Approach to Classical Music Performance: An Empirical Investigation into Its Characteristics and Impact.» Music Performance Research 6 (2013): 1-38. Imprimé.

Donin, Nicolas. «Empirical and Historical Musicologies of Compositional Processes: Towards a Crossfertilisation." The Act of Musical Composition: Studies in the Creative Process. Ed. Dave Collins. Aldershot: Ashgate, 2012. 1-26. Imprimé.

Donin, Nicolas et François-Xavier Féron. «Tracking the Composer. Cognition in the Course of a Creative Process: Stefano Gervasoni and the Beginning of Gramigna.» Musicae Scientae 16.3 (2012): 262285. Imprimé.

Dudas, Richard. «Comprovisation: The Various Facets of Composed Improvisation within Interactive Performance Systems.» Leonardo Music Journal 20 (2010): 29-31. Imprimé.

Globokar, Vinko. «lls improvisent... Improvisez... Improvisons.» Musique en jeu 6 (1971): 13-9. Imprimé.

Goehr, Lydia. The Imaginary Museum of Musical Works. Oxford: Oxford UP, 2007. Imprimé.

Guionnet, Jean-Luc. «Buttes-témoins.» Filigrane 8 (2008): 129-148. Imprimé. 
Kinderman, William. The Creative Process in Music from Mozart to Kurtág. Urbana: U of Illinois P, 2012. Imprimé.

Levaillant, Denis. L'improvisation musicale. Arles: Actes Sud, 1996. Imprimé.

Levinson, Jerrold. «Hybrid Art Forms.» Journal of Aesthetic Eduction 18 (1984): 5-13. Imprimé.

Lewis, George. «Improvised Music After 1950: Afrological and Eurological Perspectives.» Black Music Research Journal 16.1 (1996): 91-122. Imprimé.

Nettl, Bruno. «Thoughts on Musical Improvisation: A Comparative Approach.» The Musical Quarterly 60.1 (1974): 1-19. Imprimé.

Pohjannoro, Ulla. «Inspiration and Decision-making: A Case Study of a Composer's Intuitive and Reflective Thoughts.» Musicae Scientiae 18.2 (2014): 166-188. Imprimé.

Rose, Simon et Raymond MacDonald. «Improvisation as Real-Time Composition.» The Act of Musical Composition: Studies in the Creative Process. Ed. Dave Collins. Aldershot: Ashgate, 2012. 187-214. Imprimé.

Sève, Bernard. L'instrument de musique: une étude philosophique. Paris: Le Seuil, 2013. Imprimé.

Walter, Thibault. La Free Music comme mouvement et comme langage. Paris: Mémoire de la classe d'esthétique de Christian Accaoui, CNSMDP, 2006. Imprimé. 